\title{
METASTABILITY FOR PARABOLIC EQUATIONS WITH DRIFT: PART II. THE QUASILINEAR CASE
}

\author{
HITOSHI ISHII ${ }^{1, *}$ AND PANAGIOTIS E. SOUGANIDIS ${ }^{2}$
}

\begin{abstract}
This is the second part of our series of papers on metastability results for parabolic equations with drift. The aim is to present a self contained study, using partial differential equations methods, of the metastability properties of quasi-linear parabolic equations with a drift and to obtain results similar to those in Freidlin and Koralov [6, 8 .
\end{abstract}

\begin{abstract}
NotAtion. We work in $\mathbb{R}^{n}$ and write $\mathbb{S}^{n}$ for the space of real $n \times n$ symmetric matrices. For any $\theta \in(0,1], \mathbb{S}^{n}(\theta)$ denotes the subset of all $a \in \mathbb{S}^{n}$ satisfying $\theta I \leq a \leq \theta^{-1} I$, where $I$ is the $n \times n$ identity matrix. If $a \in \mathbb{S}^{n}$, then $\operatorname{tr} a$ denotes its trace, and, for $a, b \in \mathbb{S}^{n}$, $a \leq b$ if and only if $b-a$ is a nonnegative matrix. Given $p \in \mathbb{R}^{n}$, let $p \otimes p$ denote the symmetric matrix $\left(p_{i} p_{j}\right)_{1 \leq i, j \leq n}$. If $U$ is a subset of $\mathbb{R}^{k}$ for some $k \in \mathbb{N}$, then $C\left(U ; \mathbb{S}^{n}(\theta)\right)$ is the set of $\mathbb{S}^{n}(\theta)$-valued continuous maps from $U$ into $\mathbb{S}^{n}$. For $a \in \mathbb{S}^{n}$ and $p \in \mathbb{R}^{n}$, $a p \cdot p:=\sum_{i, j=1}^{n} a_{i j} p_{j} p_{i}$. If $r_{1}, r_{2} \in \mathbb{R}$, then $r_{1} \wedge r_{2}:=\min \left\{r_{1}, r_{2}\right\}$ and $r_{1} \vee r_{2}:=\max \left\{r_{1}, r_{2}\right\}$ and, for $r \in \mathbb{R}, r_{+}=r \vee 0$ and $r_{-}=(-r) \vee 0$. We use the convention $\inf \emptyset=\infty$ and $\sup \emptyset=-\infty$. The open ball in $\mathbb{R}^{n}$ with radius $R>0$ and center at $x \in \mathbb{R}^{n}$ is $B_{R}(x)$, and $B_{R}:=B_{R}(0)$. Given $\Omega \subset \mathbb{R}^{n}$ and $\delta>0$, we write $\Omega_{\delta}:=\{x \in \Omega: \operatorname{dist}(x, \partial \Omega) \geq \delta\}$, and, for $T>0, Q_{T}:=\Omega \times(0, T)$; if $T=\infty$, then we write $Q$ instead of $Q_{\infty}$. The parabolic boundary of $Q_{T}$ is $\partial_{\mathrm{p}} Q_{T}:=(\bar{\Omega} \times\{0\}) \cup(\partial \Omega \times(0, T))$. We denote by $\operatorname{Lip}\left(A, \mathbb{R}^{k}\right)$ the set of the $\mathbb{R}^{k}$-valued Lipschitz continuous functions defined in $A \subset \mathbb{R}^{k}$; when $k=1$, we often write $\operatorname{Lip}(A)$. We write $\operatorname{USC}(A)$ and $\operatorname{LSC}(A)$ for the set of, respectively, upper and lower semicontinuous functions defined on $A$, and, when $A \subset \mathbb{R}^{n} \times[0, \infty)$ is open, $C^{2,1}(A)$ is the space of functions which are continuously differentiable twice with respect to the space variables and once with respect to the time variable. Given a bounded family of functions $f_{\delta}: A \rightarrow \mathbb{R}, \limsup _{\delta \rightarrow 0}^{\star} f_{\delta}(x):=\lim _{r \rightarrow 0} \sup \left\{f_{\delta}(x+y): x+y \in A,|y|+\delta \leq r\right\}$ and $\liminf \inf _{\star \delta \rightarrow 0} f_{\delta}(x):=\lim _{r \rightarrow 0} \inf \left\{f_{\delta}(x+y): x+y \in A,|y|+\delta \leq r\right\}$. If $A$ is a closed subset of $\mathbb{R}^{n}$ and $f: A \rightarrow \mathbb{R}$, arg $\min (f \mid A):=\left\{x \in A: f(x)=\min _{y \in A} f(y)\right\}$. We use $C$ to denote constants, which may change from line to line. When we want to display the dependence of a constant $C$ on a parameter $a$, we write $C=C(a)$, and, for $a, b \in \mathbb{R}, a \approx b$ means that $a$ and $b$ are close to each other in a controlled way. Finally to simplify the notation we write $\left\{a_{k}\right\}$ to denote the sequence $\left\{a_{k}\right\}_{k \in \mathbb{N}}$.
\end{abstract}

Date: July 30, 2021.

2010 Mathematics Subject Classification. Primary 35B40; Secondary 35K20, 37H99.

Key words and phrases. parabolic equation, asymptotic behavior, metastability, stochastic perturbation.

* Corresponding author.

1 Faculty of Education and Integrated Arts and Sciences, Waseda University, Nishi-Waseda, Shinjuku, Tokyo 169-8050, Japan. Partially supported by grants, the JSPS, KAKENHI \#26220702, \#23340028 and \#23244015.

${ }^{2}$ Department of Mathematics, The University of Chicago, 5734 S. University Avenue, Chicago, IL 60657, USA. Partially supported by the National Science Foundation grant DMS-1266383. 


\section{INTRODUCTION}

This is the second part of our series of papers on metastability results for parabolic equations with drift. The aim is to present a self contained study, using partial differential equations (pde for short) methods, of the metastability properties of quasi-linear parabolic equations with a drift and to obtain results similar to those in Freidlin and Koralov [6, 8,

More precisely we are interested in the asymptotic behavior, as $\varepsilon \rightarrow 0$ and $t \rightarrow \infty$, of the solution $u^{\varepsilon}=u^{\varepsilon}(x, t)$ of the initial-boundary value problem

$$
u_{t}^{\varepsilon}=\varepsilon \operatorname{tr}\left[a\left(x, u^{\varepsilon}\right) D^{2} u^{\varepsilon}\right]+b(x) \cdot D u^{\varepsilon} \text { in } Q,
$$

and

$$
u^{\varepsilon}=g \text { on } \partial_{\mathrm{p}} Q
$$

where

$$
\Omega \text { is a bounded } C^{1} \text {-domain with outward normal vector } \nu
$$

and

$$
g \in C(\bar{\Omega}) .
$$

Throughout the paper we assume that, for some $\theta_{0} \in(0,1]$,

$$
a \in C\left(\bar{\Omega} \times \mathbb{R} ; \mathbb{S}^{n}\left(\theta_{0}\right)\right),
$$

and

$$
b \in \operatorname{Lip}\left(\mathbb{R}^{n} ; \mathbb{R}^{n}\right) \text { with } b(0)=0
$$

is such that

the origin is a (unique) globally asymptotically stable point of the dynamical system $\dot{X}=b(X)$ generated by $b$.

This last assumption is further quantified by the additional requirements that $b$ points inward at the boundary points of $\Omega$, that is,

$$
b \cdot \nu<0 \text { on } \partial \Omega
$$

and there exist $b_{0}>0$ and $r_{0}>0$ such that $\bar{B}_{r_{0}} \subset \Omega$, and

$$
b(x) \cdot x \leq-b_{0}|x|^{2} \quad \text { for all } \quad x \in B_{r_{0}} .
$$

For later use we summarize all the above assumptions in the list

$$
\text { (1.3), (1.4), (1.5), (1.6), (1.7), (1.8) and (1.9). }
$$

The asymptotic behavior of the $u^{\varepsilon}$ 's is described in Theorem 1 . Our arguments are based entirely on pde methods and the main tools are the comparison principle and the construction of two kinds of barrier functions for parabolic equations. The later was the main subject of our previous paper [11].

We work with either classical or viscosity solutions depending on the context and most of the times we say solution without making a distinction. When we write inequalities for viscosity sub- or super-solutions, we use the $\leq$ and $\geq$ signs for a sub- and super-solutions respectively. Finally, we will always work with $\varepsilon \in(0,1)$ and we will not repeat this.

An important tool is the quasi-potential $V^{c}$ associated, for each $c \in \mathbb{R}$, with $(a(\cdot, c), b)$, which is characterized by the property

$$
V^{c} \text { is the maximal subsolution of } H^{c}(x, D u)=0 \text { in } \Omega \text { and } u(0)=0 \text {, }
$$


where the Hamiltonian $H^{c} \in C\left(\bar{\Omega} \times \mathbb{R}^{n}\right)$ is given by

$$
H^{c}(x, p):=a(x, c) p \cdot p+b(x) \cdot p .
$$

The quasi-potential $V^{c}$ is also the unique (viscosity) solution $u \in \operatorname{Lip}(\bar{\Omega})$ of the stateconstraints problem for the Hamilton-Jacobi equation $H(x, D u)=0$ in $\Omega$, with the additional condition that $u(0)=0$. (See Lemma C.1 in Appendix C for the uniqueness of this state-constraints problem, and also Soner [14], Fleming and Soner [5] and Ishii [10] for some related aspects.)

Next we introduce some terminology and introduce some additional notation and hypotheses similar to those in [6, 8].

Consider the map $M: \mathbb{R} \rightarrow \mathbb{R}$ given by

$$
M(c):=\min _{\partial \Omega} V^{c} .
$$

The continuity of $a$ and the stability properties of viscosity solutions yield that the functions $\mathbb{R} \ni c \mapsto M(c)$ and $\bar{\Omega} \times \mathbb{R} \ni(x, c) \mapsto V^{c}(x) \in \mathbb{R}$ are continuous. The continuity of the latter is an easy consequence of the uniqueness of the state constraints problem.

Given $g \in C(\bar{Q})$, we set

$$
c_{0}:=g(0), \quad g_{\min }:=\min _{\bar{\Omega}} g, \quad g_{\max }:=\max _{\bar{\Omega}} g, \quad g_{1}:=\min _{\partial \Omega} g, \quad g_{2}:=\max _{\partial \Omega} g,
$$

and note that $\left[g_{1}, g_{2}\right] \subset\left[g_{\min }, g_{\max }\right]$. Henceforth we write

$$
I_{g}:=\left[g_{\min }, g_{\max }\right],
$$

and we introduce the multi-valued map $G: I_{g} \rightarrow 2^{\mathbb{R}}$ by

$$
G(c):=\left\{g(x): x \in \arg \min \left(V^{c} \mid \partial \Omega\right)\right\} .
$$

It is immediate that $G(c) \subset I_{g}$ for all $c \in I_{g}$. Moreover, since $(c, x) \mapsto V^{c}(x)$ and $g$ are continuous on $\mathbb{R} \times \partial \Omega$ and $\partial \Omega$ respectively, it is easily checked that $G$ is upper semicontinuous on $I_{g}$ and, hence, $G(c)$ is compact for all $c \in I_{g}$.

Next we define the functions $G^{ \pm}: I_{g} \rightarrow I_{g}$ by

$$
G^{+}(c):=\max G(c) \quad \text { and } \quad G^{-}(c):=\min G(c) .
$$

and note that they are respectively upper and lower semicontinuous, and, moreover,

$$
G^{+}(c)=\max _{\arg \min \left(V^{c} \mid \partial \Omega\right)} g \text { and } G^{-}(c)=\min _{\arg \min \left(V^{c} \mid \partial \Omega\right)} g .
$$

Following 6, 8, we assume that

$$
G^{+}\left(c_{0}\right)=G^{-}\left(c_{0}\right)
$$

and set

$$
g_{0}:=G^{+}\left(c_{0}\right)=G^{-}\left(c_{0}\right) .
$$

This assumption means that the set $G\left(c_{0}\right)$ is a singleton, that is,

$$
g(x)=g_{0} \quad \text { for all } x \in \arg \min \left(V^{c_{0}} \mid \partial \Omega\right) .
$$

Next we define $c_{1}$ as follows:

$$
\left\{\begin{array}{l}
\text { if } g_{0} \geq c_{0}, \text { then } c_{1}:=\inf \left\{c \in\left[c_{0}, \infty\right): G^{-}(c) \leq c\right\}, \text { and, } \\
\text { if } g_{0} \leq c_{0}, \text { then } c_{1}:=\sup \left\{c \in\left(-\infty, c_{0}\right]: G^{+}(c) \geq c\right\}
\end{array}\right.
$$



that

Note that, since $G\left(I_{g}\right):=\bigcup_{c \in I_{g}} G(c) \subset\left[g_{1}, g_{2}\right]$, we always have $c_{1} \in\left[g_{1}, g_{2}\right]$ and observe

$$
\left\{\begin{array}{l}
\text { if } c_{1}>c_{0}, \text { then } G^{-}(c)>c \text { for all } c \in\left[c_{0}, c_{1}\right), \\
\text { if } c_{1}<c_{0}, \text { then } G^{+}(c)<c \text { for all } c \in\left(c_{1}, c_{0}\right] .
\end{array}\right.
$$
is

We assume that the graph of $G$ crosses the diagonal from the left to the right at $c_{1}$, that

$$
\left\{\begin{aligned}
& \text { for all } \delta_{0}>0, \text { there exists } \delta \in\left(0, \delta_{0}\right] \text { such that } \\
& \text { if } c_{0} \geq c_{1}>g_{\min }, \text { then } G^{-}\left(c_{1}-\delta\right)>c_{1}-\delta, \\
& \text { if } c_{0} \leq c_{1}<g_{\max }, \text { then } G^{+}\left(c_{1}+\delta\right)<c_{1}+\delta
\end{aligned}\right.
$$

and we define the function $\bar{c}:(0, \infty) \rightarrow I_{g}$ as follows: For each $\lambda \in(0, \infty)$,

$$
\bar{c}(\lambda):=\left\{\begin{array}{l}
c_{0} \text { if either } \lambda<M\left(c_{0}\right) \text { or } c_{1}=c_{0}, \\
\min \left(c_{1}, \inf \left\{c \in\left[c_{0}, c_{1}\right]: M(c)=\lambda\right\}\right) \text { if } \lambda \geq M\left(c_{0}\right) \text { and } c_{1}>c_{0}, \\
\max \left(c_{1}, \sup \left\{c \in\left[c_{1}, c_{0}\right]: M(c)=\lambda\right\}\right) \text { if } \lambda \geq M\left(c_{0}\right) \text { and } c_{1}<c_{0} .
\end{array}\right.
$$

For later use we summarize the above assumptions in the list

$$
\text { (1.12) and (1.15). }
$$

Since the definition of $\bar{c}(\lambda)$ is cumbersome, for clarity and to compare with the linear problem, we discuss what happens when $a(x, c)$ is independent of $c$. In this case the quasipotential $V$ and, hence, its minimum value $M=\min _{\partial \Omega} V$ do not depend on $c$, and the multivalued map $G$ is a constant. Assumption (1.12) then states that $g_{0}=\min _{\arg \min (V \mid \partial \Omega)} g=$ $\max _{\arg \min (V \mid \partial \Omega)} g$ and $G(c)=\left\{g_{0}\right\}$ and $G^{-}(c)=G^{+}(c)=g_{0}$ for all $c \in I_{g}$. It is easily checked that, if $g(0)=g_{0}$, then $\bar{c}(\lambda)=g(0)=g_{0}$ for all $\lambda>0$, and, if either $g(0)<g_{0}$ or $g(0)>g_{0}$,

$$
\bar{c}(\lambda)= \begin{cases}c_{0} & \text { if } \lambda \leq M, \\ c_{1} & \text { if } \lambda>M,\end{cases}
$$

while, if $g(0) \neq g_{0}$, then $\bar{c}(\lambda)$ is discontinuous at $\lambda=M$.

The main result, which is similar to [6. Theorem $3.1 ; 8$, is:

Theorem 1. Assume (1.10) and (1.17) and let $\lambda>0$ be a point of continuity of $\bar{c}$. If, for $\varepsilon \in(0,1), u^{\varepsilon} \in C(\bar{Q}) \cap C^{2,1}(Q)$ is a solution of (1.1) and (1.2), then, for each $\delta>0$ so that $\Omega_{\delta} \neq \emptyset$,

$$
\lim _{\varepsilon \rightarrow 0} u^{\varepsilon}(\cdot, \exp (\lambda / \varepsilon))=\bar{c}(\lambda) \text { uniformly in } \Omega_{\delta} .
$$

In view of the previous discussion, when $a(x, c)$ is independent of $c$, that is for linear equations, Theorem 1 is the same as [11, Theorem 1], except if $g(0)=g_{0}$. In this case, [11, Theorem 1] asserts, in addition, the uniform convergence of $u^{\varepsilon}(\cdot, \exp (\lambda / \varepsilon))$ on any compact subset of $\Omega \cup \arg \min (V \mid \partial \Omega)$.

As in [6, 8], to prove Theorem 1 we need to show the following three propositions, which were proved in [8] using large deviation results from [9]. The first two together state [8, Lemma 3.11], while the third is an observation which is very crucial for the proof of Lemma 6 (see [8, Lemma 3.12]). 
Proposition 2. Assume (1.10) and let $u^{\varepsilon} \in C(\bar{Q}) \cap C^{2,1}(Q)$ be a solution of (1.1). Assume furthermore that the $u^{\varepsilon}$ 's are bounded on $Q$ uniformly on $\varepsilon$ and suppose that there exist sequences $\left\{\mu_{k}\right\},\left\{\lambda_{k}\right\} \subset(0, \infty)$ and $\left\{\varepsilon_{k}\right\} \subset(0,1)$ and constants $0<a_{1}<a_{2}$ and $\beta_{1}, \beta_{2} \in \mathbb{R}$ such that $\lim _{k \rightarrow \infty} \varepsilon_{k}=0$, and, for all $k \in \mathbb{N}$,

$$
0<a_{1} \leq \mu_{k}<\lambda_{k} \leq a_{2}, \quad u^{\varepsilon_{k}}\left(0, \exp \left(\mu_{k} / \varepsilon_{k}\right)\right)=\beta_{1} \quad \text { and } \quad u^{\varepsilon_{k}}\left(0, \exp \left(\lambda_{k} / \varepsilon_{k}\right)\right)=\beta_{2} .
$$

If $\beta_{1} \neq \beta_{2}$, then $\lim \sup _{k \rightarrow \infty} \lambda_{k} \geq M\left(\beta_{2}\right)$.

Proposition 3. Assume (1.10) and let $u^{\varepsilon} \in C(\bar{Q}) \cap C^{2,1}(Q)$ be a solution of (1.1) and (1.2). Assume further that there exist sequences $\left\{\mu_{k}\right\},\left\{\lambda_{k}\right\} \subset(0, \infty)$ and $\left\{\varepsilon_{k}\right\} \subset(0,1)$ and constants $0<a_{1}<a_{2}$ and $\beta_{1}, \beta_{2} \in I_{g}$ such that $\lim _{k \rightarrow \infty} \varepsilon_{k}=0$, and, for all $k \in \mathbb{N}$,

$$
0<a_{1} \leq \mu_{k}<\lambda_{k} \leq a_{2}, \quad u^{\varepsilon_{k}}\left(0, \exp \left(\mu_{k} / \varepsilon_{k}\right)\right)=\beta_{1} \quad \text { and } \quad u^{\varepsilon_{k}}\left(0, \exp \left(\lambda_{k} / \varepsilon_{k}\right)\right)=\beta_{2} .
$$

If $\beta_{1}<\beta_{2}$, then $G^{+}\left(\beta_{2}\right) \geq \beta_{2}$, and, if $\beta_{2}<\beta_{1}$, then $G^{-}\left(\beta_{2}\right) \leq \beta_{2}$.

Proposition 4. Assume (1.10) and let $u^{\varepsilon} \in C(\bar{Q}) \cap C^{2,1}(Q)$ be a solution of (1.1) and (1.2). Fix $\beta_{0} \in I_{g}$ and $\rho_{0}>0$, and assume that, for any $\delta>0$, there exist $\gamma>0$ and $a$ sequence $\left\{\varepsilon_{k}\right\} \subset(0,1)$ such that $\lim _{k \rightarrow \infty} \varepsilon_{k}=0$ and, for all $\rho \in\left[\rho_{0}-\gamma, \rho_{0}+\gamma\right]$ and $k \in \mathbb{N}$,

$$
u^{\varepsilon_{k}}\left(0, \exp \left(\rho / \varepsilon_{k}\right)\right) \in\left[\beta_{0}-\delta, \beta_{0}+\delta\right] \text {. }
$$

If either

$$
G^{-}\left(\beta_{0}\right)>\beta_{0} \quad \text { or } \quad G^{+}\left(\beta_{0}\right)<\beta_{0}
$$

then $\rho_{0} \leq M\left(\beta_{0}\right)$.

We discuss next some of the new ideas that are needed to prove the main theorem.

Recall that we are interested in the asymptotic behavior, as $(\varepsilon, t) \rightarrow(0, \infty)$, of the solution $u^{\varepsilon}$ of (1.1) and (1.2) in a logarithmic time scale, that is, in the behavior, as $\varepsilon \rightarrow 0$, of $u^{\varepsilon}(x, \exp (\lambda / \varepsilon))$ for any fixed $\lambda>0$. It turns out that this is a consequence of what we call "uniform asymptotic constancy" which yields that, as $t \rightarrow \infty, u^{\varepsilon}(\cdot, t)$ behaves similarly to $u^{\varepsilon}(0, t)$ in the space $C(\Omega)$ equipped with the locally uniform convergence topology,

The uniform asymptotic constancy (see Theorem 10 below) is a crucial observation that goes beyond [11. Roughly it says that, if $u^{\varepsilon}$ is a bounded solution of (2.1) , then, as $\varepsilon \rightarrow 0$, for any compact $K \subset \Omega$ and $\delta>0$,

$$
u^{\varepsilon}(x, t) \approx u^{\varepsilon}(0, t) \text { uniformly for }(x, t) \in K \times\left[\mathrm{e}^{\delta / \varepsilon}, \infty\right) .
$$

With this fact at hand the main theorem (Theorem 11) is an easy consequence of Propositions 2, 3 and 4 .

Their proofs are based on the comparison (or maximum) principle and, thus, on the construction of barriers, that is sub- and super-solutions of (1.1). We have already built such functions in our previous work [11, where the matrix $a(x, c)$ is independent of $c$. Here (see Proposition 13 and Corollary 14) we modify the construction of one class of barrier functions in order to make the comparison argument straightforward.

The building block of the barrier functions in [11] and here is viscosity solutions of $H_{\alpha}(x, D u)=0$ with some additional normalization conditions, where $\alpha \in C\left(\bar{\Omega} ; \mathbb{S}^{n}\left(\theta_{0}\right)\right)$ is is selected as explained below and $H_{\alpha}(x, p):=\alpha(x) p \cdot p+(x) \cdot p$. An important observation is that, if $V_{\alpha}$ is the quasi-potential associated with $(\alpha, \bar{b})$, then $V_{\alpha}>0$ in $\bar{\Omega} \backslash\{0\}$ and $M_{\alpha}:=\min _{\partial \Omega} V_{\alpha}>0$. 
The barriers $w^{\varepsilon}: \bar{Q} \rightarrow \mathbb{R}$ are supersolutions of (1.1) of the form

$$
w^{\varepsilon}(x, t):=\exp \left(\frac{v(x)-m}{\varepsilon}\right)+d_{\varepsilon} t
$$

where $m$ and $d_{\varepsilon}$ are positive constants such that $0<m<M_{\alpha}$ and $d_{\varepsilon}=\exp \left(-\lambda_{\varepsilon} / \varepsilon\right)$ for some $\lambda_{\varepsilon} \approx m$, and $v$ is an appropriately chosen smooth approximation of $V_{\alpha}$. The choice of $m$ yields that, for $\varepsilon$ sufficiently small, $w^{\varepsilon}$ is compatible with the Dirichlet data $g$ on $\partial \Omega \times[0, \infty)$.

In view of the fact that a priori we have little knowledge of the uniform in $\varepsilon$ regularity of solutions of (1.1), given such a solution $u^{\varepsilon}$, we treat $a^{\varepsilon}=a\left(x, u^{\varepsilon}(x, t)\right)$ as an arbitrary element of $C\left(\bar{Q} ; \mathbb{S}^{n}\left(\theta_{0}\right)\right)$.

To motivate the choice of $\alpha$ in the construction of the barrier function given the $a^{\varepsilon}$ above we compute in $Q$

$$
\begin{aligned}
w_{t}^{\varepsilon} & -\varepsilon \operatorname{tr}\left[a^{\varepsilon}(x, t) D^{2} w^{\varepsilon}\right]-b \cdot D w^{\varepsilon} \\
& =d_{\varepsilon}-\varepsilon^{-1} \exp \left(\frac{v(x)-m}{\varepsilon}\right)\left(H_{\varepsilon}(x, t, D v)+\varepsilon \operatorname{tr}\left[a^{\varepsilon} D^{2} v\right]\right)
\end{aligned}
$$

with $H_{\varepsilon}(x, t, p):=a^{\varepsilon}(x, t) p \cdot p+b(x) \cdot p$.

If $\alpha \in C\left(\bar{\Omega} ; \mathbb{S}^{n}\left(\theta_{0}\right)\right)$ satisfies $a^{\varepsilon} \leq \alpha$ in $Q$, then

$$
w_{t}^{\varepsilon}-\varepsilon \operatorname{tr}\left[a^{\varepsilon}(x, t) D^{2} w^{\varepsilon}\right]-b \cdot D w^{\varepsilon} \geq d_{\varepsilon}-\varepsilon^{-1} \exp \left(\frac{v(x)-m}{\varepsilon}\right)\left(H_{\alpha}(x, D v)+O(\varepsilon)\right) \geq 0,
$$

with the last the inequality holding, if $\varepsilon$ is sufficiently small, because of the choice of $v$ and $d_{\varepsilon}$-the details are given in Proposition 12.

A very important fact in our analysis (see Proposition 11 below for the precise statement) is that the locally uniform convergence topology of $C(\Omega)$ is strong enough to imply that, if $\alpha(x) \approx a(x, c)$ in $C(\Omega)$, then $M_{\alpha} \approx M(c)$ and $\arg \min \left(V_{\alpha} \mid \partial \Omega\right) \approx \arg \min \left(V^{c} \mid \partial \Omega\right)$.

To describe the idea which is in the core of the proof of, for example, Proposition 2, we consider the very special case that, for $\varepsilon>0$ sufficiently small and some constants $c, \gamma>0$ and $0<\delta<\mu<\lambda$,

$$
\left|u^{\varepsilon}(0, t)-c\right|<\gamma \text { for all } t \in[\exp (\delta / \varepsilon), \exp (\lambda / \varepsilon)],
$$

and

$$
u^{\varepsilon}(0, \exp (\delta / \varepsilon))=c \quad \text { and } \quad u^{\varepsilon}(0, \exp (\mu / \varepsilon))>c+\eta \text { for some } \eta \in(0, \gamma) .
$$

We then choose $\alpha \in C\left(\bar{\Omega} ; \mathbb{S}^{n}\left(\theta_{0}\right)\right)$ so that $a^{\varepsilon} \leq \alpha$ in $\Omega \times\left[t_{\varepsilon}, T_{\varepsilon}\right]$, where $t_{\varepsilon}:=\exp (\delta / \varepsilon)$ and $T_{\varepsilon}:=\exp (\lambda / \varepsilon)$. Using the barrier $w^{\varepsilon}$ as in the linear case (see [11, Theorem 1 (i)]), we conclude that, as $\varepsilon \rightarrow 0$, for any $\rho<M_{\alpha}, u^{\varepsilon}(0, t) \rightarrow c$ for all $t \in\left[t_{\varepsilon}, T_{\varepsilon} \wedge \exp (\rho / \varepsilon)\right]$, which implies that $\mu \geq M_{\alpha}$. Furthermore, according to the previous arguments, $\alpha$ can be chosen, so that, as $\gamma \rightarrow 0, M_{\alpha} \rightarrow M^{c}$.

Organization of the paper. The rest of the paper is organized as follows. In Section 2 we study the asymptotic constancy, that is the effect of the drift term in parabolic equations like (1.1). In Section 3 we introduce Hamilton-Jacobi equations related to (1.1), which have quadratic nonlinearity, and study the continuity properties of the associated quasipotentials. Section 4 is devoted to the construction of two kind of barrier functions, or sub- and super-solutions, which are used to study the asymptotic behavior of solutions of linear parabolic equations, that is equations like (1.1) with $a \in C\left(\bar{Q} ; \mathbb{S}^{n}\left(\theta_{0}\right)\right)$. The proofs 
of Propositions 2, 3 and 4 and Theorem 1 are given in Sections 5 and 6 respectively. Some basic properties of viscosity solutions are explained in Appendices A, B and C.

\section{The Asymptotic constancy}

We consider the linear pde

$$
u_{t}^{\varepsilon}=\varepsilon \operatorname{tr}\left[a^{\varepsilon}(x, t) D^{2} u^{\varepsilon}\right]+b(x) \cdot D u^{\varepsilon} \quad \text { in } Q .
$$

We assume, in addition to (1.6) and (1.9), that

$$
a^{\varepsilon} \in C\left(\bar{Q}, \mathbb{S}^{n}\left(\theta_{0}\right)\right) \text {. }
$$

The goal here is to show that the drift term in (2.1) has a strong effect to propagate, as $\varepsilon \rightarrow 0$, the values of the solutions $u^{\varepsilon}$ at $x=0$ to $\Omega$; for future reference we call this fact the asymptotic constancy.

It turns out that the asymptotic constancy does not depend on any properties of $a^{\varepsilon}$ other than (2.2). It is, therefore, technically more convenient to study, in some instances, instead of (2.1), the problem

$$
v_{t}=\varepsilon P^{+}\left(D^{2} v\right)+b(x) \cdot D v \quad \text { in } Q,
$$

where $P^{+}$is the Pucci operator associated with $\mathbb{S}^{n}\left(\theta_{0}\right)$ defined by

$$
P^{+}(X)=\sup \left\{\operatorname{tr}[A X]: A \in \mathbb{S}^{n}\left(\theta_{0}\right)\right\},
$$

which is, obviously, uniformly elliptic with constants $\theta_{0}$ and $\theta_{0}^{-1}$, that is, for all matrices $X, Y \in \mathbb{S}^{n}$ such that $X \leq Y$,

$$
\theta_{0} \operatorname{tr}(Y-X) \leq P^{+}(Y)-P^{+}(X) \leq \theta_{0}^{-1} \operatorname{tr}(Y-X) .
$$

Some useful barrier functions. We fix an auxiliary function $h \in C^{2}([0, \infty))$ with the properties

$$
0 \leq h \leq 1, h=0 \text { in }[0,1 / 2], h=1 \text { in }[1, \infty) \text { and } h^{\prime} \geq 0
$$

set

$$
k:=b_{0} / 2 \quad \text { and } \quad R_{0}:=2 \sqrt{2 n} / \sqrt{b_{0} \theta_{0}},
$$

choose $R \in\left[R_{0}, \infty\right), r \in\left(0, r_{0}\right]$, where $r_{0}$ is as in (1.9), and $\varepsilon_{0} \in(0,1)$ so that

$$
\sqrt{\varepsilon_{0}} R<r,
$$

and, for $\varepsilon \in\left(0, \varepsilon_{0}\right]$, let

$$
\tau=\tau(\varepsilon):=\frac{1}{k} \log \left(\frac{r}{R \sqrt{\varepsilon}}\right) .
$$

With all these choices at hand we introduce the functions $p^{\varepsilon}, q^{\varepsilon}: \mathbb{R}^{n} \times[0, \infty) \rightarrow \mathbb{R}$ defined by

$$
p^{\varepsilon}(x, t):=h\left((R \sqrt{\varepsilon})^{-1}|x| \mathrm{e}^{-k t}\right)
$$

and

$$
q^{\varepsilon}(x, t):=p^{\varepsilon}(x, t)+\frac{\left\|h^{\prime \prime}\right\|_{L^{\infty}}}{R^{2} \theta_{0}} \int_{0}^{t} \mathrm{e}^{-2 k s} \mathrm{~d} s ;
$$

observe that, since $h$ vanishes identically in a neighborhood of the positive time axis $l:=$ $\{0\} \times(0, \infty), p^{\varepsilon}$ and $q^{\varepsilon}$ are smooth in $\mathbb{R}^{n} \times(0, \infty)$. 
We note that $p^{\varepsilon}$ appears in the proof of $[6$, Lemma $3.6 ; 8$. The difference is that these references consider equations like (2.1), while here we study (2.3).

The following lemma summarizes the properties of $q^{\varepsilon}$. Its proof is based on long explicit but also straightforward calculations. The reader may want to skip the details on first reading.

Lemma 1. Assume (1.6), (1.9) and (2.5). With the above choices of $k, R, r, \varepsilon_{0}, \varepsilon$ and $\tau$, the function $q^{\varepsilon}$ given by (2.10) is a supersolution to (2.3) in $B_{r_{0}} \times(0, \infty)$. Moreover,

$$
\left\{\begin{array}{l}
q^{\varepsilon}(\cdot, 0) \geq 0 \text { in } B_{r}, \quad q^{\varepsilon}(\cdot, 0) \geq 1 \quad \text { in } B_{r} \backslash B_{\sqrt{\varepsilon} R} \\
q^{\varepsilon} \geq 1 \text { in } \partial B_{r} \times[0, \tau] \text { and } q^{\varepsilon}(\cdot, \tau) \leq \frac{\left\|h^{\prime \prime}\right\|_{L^{\infty}}}{b_{0} \theta_{0} R^{2}} \text { on } B_{r / 2} .
\end{array}\right.
$$

Proof. First note that

$$
p^{\varepsilon}(x, t)=1 \quad \text { if } \quad|x| \geq R \sqrt{\varepsilon} \mathrm{e}^{k t} \quad \text { and } \quad p^{\varepsilon}(x, t)=0 \quad \text { if } \quad|x| \leq \frac{1}{2} R \sqrt{\varepsilon} \mathrm{e}^{k t} .
$$

For $(x, t) \in B_{r_{0}} \times(0, \infty)$ we write $\rho=\frac{1}{R \sqrt{\varepsilon}}, r_{x, t}=(R \sqrt{\varepsilon})^{-1}|x| \mathrm{e}^{-k t}$ and $\bar{x}:=x /|x|$ (since, in view of the above, $p^{\varepsilon}$ vanishes in a neighborhood of the origin we do not have to be concerned about $x=0$ ), and find

$$
\begin{aligned}
p_{t}^{\varepsilon}(x, t) & =-k h^{\prime}\left(r_{x, t}\right)|x| \rho \mathrm{e}^{-k t}, \quad D p^{\varepsilon}(x, t)=h^{\prime}\left(r_{x, t}\right) \rho \bar{x} \mathrm{e}^{-k t}, \\
D^{2} p^{\varepsilon}(x, t) & =h^{\prime}\left(r_{x, t}\right) \rho \mathrm{e}^{-k t} \frac{1}{|x|}(I-\bar{x} \otimes \bar{x})+h^{\prime \prime}\left(r_{x, t}\right) \rho^{2} \mathrm{e}^{-2 k t} \bar{x} \otimes \bar{x} .
\end{aligned}
$$

Moreover, for any $a \in \mathbb{S}^{n}\left(\theta_{0}\right)$ and all $(x, t) \in \bar{Q}$ with $x \neq 0$, we have

$$
|\operatorname{tr}[a(I-\bar{x} \otimes \bar{x})]| \leq \theta_{0}^{-1}(n-1)<\theta_{0}^{-1} n \quad \text { and } \quad|\operatorname{tr}[a \bar{x} \otimes \bar{x}]| \leq \theta_{0}^{-1},
$$

and, therefore,

$$
\begin{aligned}
p_{t}^{\varepsilon}- & \varepsilon \operatorname{tr}\left[a D^{2} p^{\varepsilon}\right]-b(x) \cdot D p^{\varepsilon} \\
= & h^{\prime}\left(r_{x, t}\right) \rho|x| \mathrm{e}^{-k t}\left\{-k-|x|^{-1} b(x) \cdot \bar{x}-\frac{\varepsilon}{|x|^{2}} \operatorname{tr}[a(I-\bar{x} \otimes \bar{x})]\right\} \\
& -\varepsilon h^{\prime \prime}\left(r_{x, t}\right) \rho^{2} \mathrm{e}^{-2 k t} \operatorname{tr}[a \bar{x} \otimes \bar{x}] \\
\geq & h^{\prime}\left(r_{x, t}\right) \rho|x| \mathrm{e}^{-k t}\left\{-k+b_{0}-\frac{n \varepsilon}{\theta_{0}|x|^{2}}\right\}-\varepsilon\left\|h^{\prime \prime}\right\|_{L^{\infty}} \rho^{2} \mathrm{e}^{-2 k t} \theta_{0}^{-1} .
\end{aligned}
$$

Observe that

$$
\frac{1}{2} \leq r_{x, t} \leq 1 \quad \text { if and only if } \frac{1}{2} R \sqrt{\varepsilon} \mathrm{e}^{k t} \leq|x| \leq R \sqrt{\varepsilon} \mathrm{e}^{k t},
$$

and

$$
h^{\prime}\left(r_{x, t}\right) \frac{1}{|x|^{2}} \leq h^{\prime}\left(r_{x, t}\right) \frac{4 \mathrm{e}^{-2 k t}}{R^{2} \varepsilon} \leq h^{\prime}\left(r_{x, t}\right) \frac{4}{R^{2} \varepsilon} .
$$

Using the observations above and (1.9) and recalling the choices of the constants and that $a \in \mathbb{S}^{n}\left(\theta_{0}\right)$ is arbitrary, we get

$$
\begin{aligned}
p_{t}^{\varepsilon} & -\varepsilon P^{+}\left(D^{2} p^{\varepsilon}\right)-b(x) \cdot D p^{\varepsilon} \\
& \geq h^{\prime}\left(r_{x, t}\right) \rho|x| \mathrm{e}^{-k t}\left\{-k+b_{0}-\frac{4 n}{\theta_{0} R^{2}}\right\}-\left\|h^{\prime \prime}\right\|_{L^{\infty}} \frac{\mathrm{e}^{-2 k t}}{\theta_{0} R^{2}} \geq-\left\|h^{\prime \prime}\right\|_{L^{\infty}} \frac{\mathrm{e}^{-2 k t}}{\theta_{0} R^{2}} .
\end{aligned}
$$


Thus, noting that, for all $t>0$,

$$
p_{t}^{\varepsilon}(0, t)-\varepsilon P^{+}\left(D^{2} p^{\varepsilon}(0, t)\right)-b(0) \cdot D p^{\varepsilon}(0, t)=0
$$

we conclude that

$$
p_{t}^{\varepsilon}-\varepsilon P^{+}\left(D^{2} p^{\varepsilon}\right)-b(x) \cdot D p^{\varepsilon} \geq-\left\|h^{\prime \prime}\right\|_{L^{\infty}} \frac{\mathrm{e}^{-2 k t}}{\theta_{0} R^{2}} \text { in } B_{r_{0}} \times(0, \infty),
$$

and, hence, $q^{\varepsilon}$ is a supersolution of (2.3) in $B_{r_{0}} \times(0, \infty)$.

Finally, we observe that, if $0 \leq t \leq \tau$ and $x \in \partial B_{r}$, then

$$
\frac{|x| \mathrm{e}^{-k t}}{\sqrt{\varepsilon} R} \geq \frac{r \mathrm{e}^{-k \tau}}{\sqrt{\varepsilon} R}=1 \quad \text { and } \quad q^{\varepsilon}(x, t) \geq p^{\varepsilon}(x, t)=1,
$$

and, if $x \in B_{r / 2}$, then

$$
\frac{|x| \mathrm{e}^{-k \tau}}{\sqrt{\varepsilon} R} \leq \frac{r \mathrm{e}^{-k \tau}}{2 \sqrt{\varepsilon} R}=\frac{1}{2} \quad \text { and } \quad q^{\varepsilon} \leq p^{\varepsilon}+\frac{\left\|h^{\prime \prime}\right\|_{L^{\infty}}}{b_{0} \theta_{0} R^{2}}=\frac{\left\|h^{\prime \prime}\right\|_{L^{\infty}}}{b_{0} \theta_{0} R^{2}} .
$$

Moreover,

$$
q^{\varepsilon}(x, 0)=p^{\varepsilon}(x, 0)=h(|x| /(\sqrt{\varepsilon} R)) \geq \begin{cases}0 & \text { for all } x \in B_{r}, \\ 1 & \text { for all } x \in B_{r} \backslash B_{\sqrt{\varepsilon} R} .\end{cases}
$$

An application of the Harnack inequality. We use a consequence of the Harnack inequality to obtain an a priori bound for the oscillations of the $u^{\varepsilon}$ 's, which are uniform in $\varepsilon$ and $t$ up to $\infty$.

If $u^{\varepsilon} \in C^{2,1}(Q)$ is a solution of (2.1), then

$$
v^{\varepsilon}(y, t):=u^{\varepsilon}(\sqrt{\varepsilon} y, t) \quad \text { for } \quad(y, t) \in B_{r_{0} / \sqrt{\varepsilon}} \times[0, \infty),
$$

satisfies

$$
v_{t}^{\varepsilon}=\operatorname{tr}\left[a^{\varepsilon}(\sqrt{\varepsilon} y, t) D^{2} v^{\varepsilon}\right]+\frac{b(\sqrt{\varepsilon} y)}{\sqrt{\varepsilon}} \cdot D v^{\varepsilon} \text { in } B_{r_{0} / \sqrt{\varepsilon}} \times(0, \infty) .
$$

It also follows from (1.6) that there exists $L_{b}>0$ such that

$$
|b(x)| \leq L_{b}|x| \text { for all } x \in B_{r_{0}},
$$

and, hence,

$$
\frac{|b(\sqrt{\varepsilon} y)|}{\sqrt{\varepsilon}} \leq L_{b}|y| \quad \text { for all } y \in B_{r_{0} / \sqrt{\varepsilon}} \text {. }
$$

Next we recall the following consequence of the Harnack inequality from Krylov [12, Theorem 4.2.1].

Proposition 5. Assume (2.2) and (2.13), fix $R \in(0,2],(z, \tau) \in \mathbb{R}^{n} \times(0, \infty)$ such that $\left.B_{R}(z) \subset B_{r_{0} / \sqrt{\varepsilon}}\right)$ and $\tau>2 R^{2}$, and let $w \in C^{2,1}\left(B_{R}(z) \times\left(\tau-2 R^{2}, \tau\right)\right)$ be a nonnegative solution of (2.12) in $B_{R}(z) \times\left(\tau-2 R^{2}, \tau\right)$. There exists a constant $C=C\left(R, \theta_{0}, L_{b}, n\right)>1$ such that

$$
w\left(z, \tau-R^{2}\right) \leq C \inf _{y \in B_{R / 2}(z)} w(y, \tau) .
$$

We use now Proposition 5 to obtain the following improvement of oscillation-type result for solutions to (2.1). 
Corollary 6. Assume (2.2) and (2.13) and, for $\varepsilon \in(0,1)$, let $u^{\varepsilon} \in C(\bar{Q}) \cap C^{2,1}(Q)$ be a solution of (2.1) in $Q$. Fix $m \in \mathbb{N}$ and $T>0$ and assume that $(m+2) \sqrt{\varepsilon} \leq r_{0}, T>4(m+1)$ and

$$
\begin{cases}u^{\varepsilon}(0, t) \leq 0 & \text { for all } t \in(0, T), \\ u^{\varepsilon}(x, t) \leq 1 & \text { for all }(x, t) \in B_{(m+2) \sqrt{\varepsilon}} \times(0, T) .\end{cases}
$$

There exists a constant $\eta=\eta\left(m, \theta_{0}, L_{b}, n\right) \in(0,1)$ such that

$$
u^{\varepsilon} \leq \eta \quad \text { in } \quad B_{m \sqrt{\varepsilon}} \times(4(m+1), T) .
$$

Proof. Noting that the function $v^{\varepsilon}(y, t)=u^{\varepsilon}(\sqrt{\varepsilon} y, t)$ is defined on $B_{m+2} \times(0, T)$, we set

$$
w(x, t)=1-v^{\varepsilon}(x, t) \quad \text { for }(x, t) \in B_{m+2} \times(0, T) .
$$

Observe that $w$ is a solution of (2.12) in $B_{m+2} \times(0, T)$ and, by (2.14), that $w$ is a nonnegative function on $B_{m+2} \times(0, T)$ and satisfies

$$
w(0, t) \geq 1 \quad \text { for all } t \in(0, T) .
$$

Let $(x, t) \in B_{m} \times(4(m+1), T)$ and choose a finite sequence of balls $B_{1}\left(x_{1}\right), \ldots, B_{1}\left(x_{m}\right) \subset$ $B_{m}$ so that $x_{1}=0, x \in B_{1}\left(x_{m}\right)$ and, if $1 \leq i<m$, then $B_{1}\left(x_{i+1}\right) \cap B_{1}\left(x_{i}\right) \neq \emptyset$. Applying Proposition 5 with $R=2$ yields, for some $C=C\left(\theta_{0}, L_{b}, n\right)>1$,

$$
w(0, t-4 m) \leq C \inf _{y \in B_{1}\left(x_{1}\right)} w(y, t-4(m-1)),
$$

and, hence, if $m=1$, we have

$$
w(0, t-4 m) \leq C^{m} w(x, t),
$$

while, if $m>1$, repeating the argument above we obtain

$$
\begin{aligned}
w(0, t-4 m) & \leq C w\left(x_{2}, t-4(m-1)\right) \leq C^{2} \inf _{y \in B_{1}\left(x_{2}\right)} w(y, t-4(m-2)) \\
& \leq \cdots \leq C^{m} \inf _{y \in B_{1}\left(x_{m}\right)} w(y, t) \leq C^{m} w(x, t) .
\end{aligned}
$$

Thus, we have $w(0, t-4 m) \leq C^{m} w(x, t)$, and, since $w(0, t-4 m) \geq 1$ by (2.14), we get

$$
1 \leq C^{m}\left(1-v^{\varepsilon}(x, t)\right)
$$

which yields

and, hence, with $\eta=1-1 / C^{m}$,

$$
v^{\varepsilon}(x, t) \leq 1-\frac{1}{C^{m}}
$$

$$
u^{\varepsilon}(x, t) \leq \eta \quad \text { for all }(x, t) \in B_{m \sqrt{\varepsilon}} \times(4(m+1), T) .
$$

The asymptotic constancy. Let $\Pi$ be a relatively open, possibly empty, subset of $\partial \Omega$, set $\Omega^{\Pi}:=\Omega \cup \Pi$, and, for any $\delta>0$,

$$
\Omega_{\delta}:=\{x \in \bar{\Omega}: \operatorname{dist}(x, \partial \Omega)>\delta\} \quad \text { and } \quad \Omega_{\delta}^{\Pi}:=\{x \in \bar{\Omega}: \operatorname{dist}(x, \partial \Omega \backslash \Pi)>\delta\} .
$$

The next result is the first indication of what we call asymptotic constancy, which is a straightforward generalization of [11, Theorem 14]. Roughly it says that, for $\varepsilon$ small, if a solution of (2.1) is bounded and small (say negative) in a small cylinder around the positive time axis $l$ and a portion of the parabolic boundary, then it is small (of order $\delta>0$ ) in a large part of $Q$ after some uniform time depending on $\delta$. 
Proposition 7. Assume (1.3), (1.6), (1.7), (1.8), (1.9) and (2.2) and fix $\delta \in\left(0, r_{0}\right)$. There exist $T_{\delta}>0$ and $\varepsilon_{0} \in(0,1)$, which depend only on $\delta, \theta_{0}, b, \Pi$ and $\Omega$, such that, if, for $\varepsilon \in\left(0, \varepsilon_{0}\right), u^{\varepsilon} \in C(\bar{Q}) \cap C^{2,1}(Q)$ is a solution of (2.1) and satisfies, for some $T(\varepsilon) \in\left(T_{\delta}, \infty\right]$,

$$
u^{\varepsilon} \leq 1 \text { in } \Omega \times[0, T(\varepsilon)) \text { and } u^{\varepsilon} \leq 0 \text { in }\left(B_{\delta} \cup \Pi\right) \times[0, T(\varepsilon)) \text {, }
$$

then

$$
u^{\varepsilon}(x, t) \leq \delta \quad \text { for all }(x, t) \in \Omega_{\delta}^{\Pi} \times\left[T_{\delta}, T(\varepsilon)\right) .
$$

For the proof of Proposition 7 it is necessary to first describe some preliminary facts that are consequence of the asymptotic stability property of the vector field $b$.

We fix $\delta>0$ and set

$$
\tau(x):=\sup \left\{t \geq 0: X(t, x) \notin B_{\delta}\right\} \quad \text { for } \quad x \in \bar{\Omega},
$$

where $X(t)=X(t, x)$ is the solution of

$$
\dot{X}(t ; x)=b(X(t ; x)) \quad \text { and } \quad X(0 ; x)=x .
$$

Since $\Omega$ is bounded and the origin is a globally asymptotically stable point of $b$, it is immediate that, if

$$
T_{\delta}:=\sup _{x \in \bar{\Omega}} \tau(x)
$$

then

$$
0<T_{\delta}<\infty \text { and } X(t, x) \in B_{\delta} \text { for all }(x, t) \in \bar{\Omega} \times\left[T_{\delta}, \infty\right) .
$$

We consider the transport problem

$$
\begin{cases}U_{t} \leq b \cdot D U & \text { in } \Omega \times\left(0, T_{\delta}\right], \\ \min \left\{U_{t}-b \cdot D U, U\right\} \leq 0 & \text { on } \Pi \times\left(0, T_{\delta}\right], \\ U \leq 0 & \text { in } B_{\delta} \times\{0\}\end{cases}
$$

the first inequality in (2.17) should be understood in the viscosity subsolution sense while the second is a viscosity interpretation of the Dirichlet condition, $U \leq 0$, on $\Pi$ (see [10]).

Lemma 2. Assume (1.3), (1.6), (1.7) and (1.8). If $U \in \operatorname{USC}\left(\bar{\Omega} \times\left[0, T_{\delta}\right]\right)$ is a subsolution of (2.17), then $U\left(x, T_{\delta}\right) \leq 0$ for all $x \in \Omega^{\Pi}$.

Proof. Fix $x \in \Omega^{\Pi}$ and, for $t \in\left[0, T_{\delta}\right]$, set

$$
u(t)=U\left(X\left(T_{\delta}-t, x\right), t\right) .
$$

It is a standard observation (see Lemma A.1 in Appendix A) that $u \in \mathrm{USC}\left(\left[0, T_{\delta}\right]\right)$ is a subsolution, if $x \in \Omega$, of

$$
u^{\prime} \leq 0 \quad \text { in }\left(0, T_{\delta}\right]
$$

and, if $x \in \Pi$, of

$$
\left\{\begin{array}{l}
u^{\prime} \leq 0 \quad \text { in }\left(0, T_{\delta}\right), \\
u^{\prime} \leq 0 \quad \text { or } \quad u \leq 0 \quad \text { on }\left\{T_{\delta}\right\} .
\end{array}\right.
$$

Suppose that $\max _{\left[0, T_{\delta}\right]} u>0$. Since $X\left(T_{\delta}, x\right) \in B_{\delta}$ and $u(0)=U\left(X\left(T_{\delta}, x\right), 0\right) \leq 0$, there must exist $\alpha>0$ and $\tau \in\left(0, T_{\delta}\right]$ such that the function $\left[0, T_{\delta}\right] \ni t \rightarrow u(t)-\alpha t$ attains its maximum on $\left[0, T_{\delta}\right]$ at $\tau$. In view of (2.18), if $x \in \Omega$, then $\alpha \leq 0$, which is a contradiction. If $x \in \Pi$, then either $\alpha \leq 0$ or $\tau=T_{\delta}$ and $u\left(T_{\delta}\right) \leq 0$, which is again a contradiction. Thus, 
we conclude that $u \leq 0$ on $\left[0, T_{\delta}\right]$. In particular, $u\left(T_{\delta}\right) \leq 0$, which shows that $U\left(x, T_{\delta}\right) \leq 0$ for all $x \in \Omega^{\Pi}$.

We proceed with the proof of Proposition 7.

Proof of Proposition 7. Let $T_{\delta}>0$ be the number defined by (2.15). For any $\varepsilon \in(0,1)$, let $\mathcal{V}_{\varepsilon}$ denote the set of all (viscosity) subsolutions $v \in \operatorname{USC}\left(\bar{\Omega} \times\left[0, T_{\delta}\right]\right)$ of $(2.3)$ such that

$$
v \leq 1 \text { on } \bar{\Omega} \times\left[0, T_{\delta}\right] \text { and } v \leq 0 \text { on }\left(B_{\delta} \cup \Pi\right) \times\left[0, T_{\delta}\right],
$$

and note that $\mathcal{V}_{\varepsilon}$, which is clearly nonempty, depends only on $\delta, T_{\delta}, \theta_{0}, b, \Pi$ and $\Omega$.

It turns out that $\mathcal{V}_{\varepsilon}$ has a maximum element. Indeed, for $(x, t) \in \bar{\Omega} \times\left[0, T_{\delta}\right]$, set

$$
v^{\varepsilon}(x, t):=\sup \left\{v(x, t): v \in \mathcal{V}_{\varepsilon}\right\}
$$

and consider its upper semicontinuous envelope

$$
\bar{v}^{\varepsilon}(x, t):=\lim _{r \rightarrow 0} \sup \left\{v^{\varepsilon}(y, s):(y, s) \in \bar{\Omega} \times\left[0, T_{\delta}\right],|(y, s)-(x, t)|<r\right\} .
$$

Standard arguments from the theory of viscosity solutions yield that $\bar{v}^{\varepsilon} \in \mathcal{V}_{\varepsilon}$ and, since $0 \in \mathcal{V}_{\varepsilon}, \bar{v}^{\varepsilon} \geq 0$ on $\bar{\Omega} \times\left[0, T_{\delta}\right]$.

Let $U \in \operatorname{USC}\left(\bar{\Omega} \times\left[0, T_{\delta}\right]\right)$ be the half-relaxed upper limit of $\bar{v}^{\varepsilon}$, that is, for $(x, t) \in$ $\bar{\Omega} \times\left[0, T_{\delta}\right]$,

$$
U(x, t):=\limsup _{\varepsilon \rightarrow 0} \bar{v}^{\varepsilon}(x, t) ;
$$

we refer to Crandall, Ishii and Lions [3] for more discussion about the half relaxed upper and lower limits.

It follows from Lemma 2 that $U\left(x, T_{\delta}\right) \leq 0$ for all $x \in \Omega^{\Pi}$, and, hence, in view of the uniformity encoded in the definition of $U$, there exists a constant $\varepsilon_{0} \in(0,1)$, depending only on $\delta, \theta_{0}, b, \Pi$ and $\Omega$, such that, for all $\varepsilon \in\left(0, \varepsilon_{0}\right)$,

$$
v^{\varepsilon}\left(\cdot, T_{\delta}\right) \leq \delta \text { on } \Omega_{\delta}^{\Pi} .
$$

Finally, since, for each $\varepsilon$, the function $\bar{\Omega} \times\left[0, T_{\delta}\right] \ni(x, t) \mapsto u^{\varepsilon}(x, s+t)$, with $0 \leq s<$ $T(\varepsilon)-T_{\delta}$, belongs to $\mathcal{V}_{\varepsilon}$, it follows that, if $s \in\left[0, T(\varepsilon)-T_{\delta}\right)$, then

$$
u^{\varepsilon}\left(x, s+T_{\delta}\right) \leq v^{\varepsilon}\left(x, T_{\delta}\right) \leq \delta \quad \text { for all } x \in \Omega_{\delta}^{\Pi} \text { and } \varepsilon \in\left(0, \varepsilon_{0}\right],
$$

and, thus,

$$
u^{\varepsilon}(x, t) \leq \delta \quad \text { for all }(x, t) \in \Omega_{\delta}^{\Pi} \times\left[T_{\delta}, T(\varepsilon)\right) \text { and } \varepsilon \in\left(0, \varepsilon_{0}\right]
$$

Next we use Corollary [6 and the previous proposition to obtain a refinement. Here we assume an upper bound, say 1, only in a cylindrical neighborhood of the positive time axis $l$ and show that, if, in addition, the solutions are small, say less than 0 on the half line $l$, then they are small, say less than $\delta$, after a time, of order $|\log \varepsilon|$, in a small cylindrical neighborhood of $l$. We remark that a time period of order $|\log \varepsilon|$ is "very short" in the logarithmic scale of time, that is, as $\varepsilon \rightarrow 0$, if $\exp \left(\lambda_{\varepsilon} / \varepsilon\right)=O(|\log \varepsilon|)$, then $\lambda_{\varepsilon} \rightarrow 0$.

Proposition 8. Assume (1.3), (1.6), (1.7), (1.9) and (2.2). For any $\delta>0$, there exist $\varepsilon_{0} \in(0,1)$ and a family $\{\tau(\varepsilon)\}_{0<\varepsilon \leq \varepsilon_{0}} \subset(0, \infty)$, both depending on $r_{0}, \theta_{0}, b, \delta$ and $n$, and $\gamma \in(0,1)$, such that, if, for $\varepsilon \in\left(0, \varepsilon_{0}\right], u^{\varepsilon}$ is a solution of (2.1) with the property that, for some $T(\varepsilon) \in(\tau(\varepsilon), \infty]$,

$$
u^{\varepsilon} \leq 1 \text { in } B_{r_{0}} \times(0, T(\varepsilon)) \text { and } u^{\varepsilon}(0, t) \leq 0 \text { for all } t \in(0, T(\varepsilon)) \text {, }
$$


then

$$
u^{\varepsilon} \leq \delta \quad \text { in } \quad B_{\gamma r_{0}} \times(\tau(\varepsilon), T(\varepsilon))
$$

Moreover, there exists a constant $C>0$, which depends on $r_{0}, \theta_{0}, b, \delta$ and $n$, such that

$$
\tau(\varepsilon) \leq C(|\log \varepsilon|+1) \quad \text { for all } \varepsilon \in\left(0, \varepsilon_{0}\right] .
$$

Although it appears similar, Proposition 8 is actually very different from [11, Theorem 13]. Indeed the second condition in (2.21) on the solutions is required only at the origin, while in [11, Theorem 13] it is assumed on a neighborhood of the origin. This refinement, which is important for the proofs of Propositions 2, 3 and 4, depends technically on the barrier functions $q^{\varepsilon}$ in Lemma 1 and the Harnack inequality (Proposition 5).

Proof of Proposition 8. To simplify the argument, we assume that $T(\varepsilon)=\infty$ since the general case can be treated similarly. that

Fix $\delta>0$, choose $h \in C^{2}([0, \infty))$ satisfying (2.6) and $m=m\left(\theta_{0}, n,\left\|h^{\prime \prime}\right\|_{L^{\infty}}\right) \in \mathbb{N}$ such

$$
\frac{\left\|h^{\prime \prime}\right\|_{L^{\infty}}}{b_{0} \theta_{0} m^{2}} \leq \frac{1}{2} \quad \text { and } \quad m \geq \frac{2 \sqrt{2 n}}{\sqrt{b_{0} \theta_{0}}}
$$

let $\eta=\eta\left(\theta_{0}, L_{b}, n\right) \in(0,1)$ be the constant in Corollary 6 set $\tau_{0}=4(m+1)$ and fix $\varepsilon_{1}=\varepsilon_{1}\left(r_{0}, m\right) \in(0,1)$ so that

$$
(m+2) \sqrt{\varepsilon_{1}} \leq r_{0}
$$

Then, for any $\varepsilon \in\left(0, \varepsilon_{1}\right]$, Corollary 6 gives

$$
u^{\varepsilon}(x, t) \leq \eta \quad \text { for all } \quad(x, t) \in B_{m \sqrt{\varepsilon}} \times\left(\tau_{0}, \infty\right) .
$$

Define

$$
v^{\varepsilon}:=(1-\eta)^{-1}\left(u^{\varepsilon}-\eta\right) \text { in } \Omega \times[0, \infty),
$$

and note that $v^{\varepsilon}$ is a solution of (2.1), and, moreover,

$$
v^{\varepsilon} \leq 1 \text { in } B_{r_{0}} \times(0, \infty) \text { and } v^{\varepsilon} \leq 0 \text { on } \bar{B}_{m \sqrt{\varepsilon}} \times\left[\tau_{0}, \infty\right) .
$$

Let $q^{\varepsilon}$ be given by (2.10) with $R$ and $r$ replaced by $m$ and $r_{0}$ respectively. It follows from Lemma 1 and the comparison principle that, for any fixed $s \geq \tau_{0}$,

$$
v^{\varepsilon}(\cdot, s+\cdot) \leq q^{\varepsilon} \text { in } B_{r_{0}} \times\left[0, \tau_{1}\right]
$$

where $\tau_{1}=\tau_{1}(\varepsilon)>0$ is given by

$$
\frac{\theta_{0} \tau_{1}}{2}=\log \left(\frac{r_{0}}{m \sqrt{\varepsilon}}\right)
$$

Hence,

$$
v^{\varepsilon}\left(\cdot, \cdot+\tau_{1}\right) \leq \frac{\left\|h^{\prime \prime}\right\|_{L^{\infty}}}{b_{0} \theta_{0} m^{2}} \leq \frac{1}{2} \text { in } B_{r_{0} / 2} \times\left[\tau_{0}, \infty\right),
$$

which, with $T_{1}(\varepsilon):=\tau_{0}+\tau_{1}(\varepsilon)$, can be rewritten as

$$
u^{\varepsilon} \leq \eta+\frac{1-\eta}{2}=\frac{1}{2}(1+\eta) \text { in } B_{r_{0} / 2} \times\left[T_{1}(\varepsilon), \infty\right) .
$$

Next, for $j=2,3, \ldots$, we choose $\varepsilon_{j} \in\left(0, \varepsilon_{j-1}\right)$ so that

$$
(m+2) \sqrt{\varepsilon_{j}} \leq \frac{r_{0}}{2^{j-1}},
$$


and, for any $\varepsilon \in\left(0, \varepsilon_{j}\right)$, select $\tau_{j}=\tau_{j}(\varepsilon)>\tau_{j-1}(\varepsilon)$ so that

$$
\frac{\theta_{0} \tau_{j}(\varepsilon)}{2}=\log \left(\frac{r_{0}}{2^{j-1} m \sqrt{\varepsilon}}\right)
$$

and set, for $\varepsilon \in\left(0, \varepsilon_{j}\right)$,

$$
T_{j}(\varepsilon):=T_{j-1}(\varepsilon)+\tau_{0}+\tau_{j}(\varepsilon)=j \tau_{0}+\sum_{i=1}^{j} \tau_{i}(\varepsilon) .
$$

We prove by induction that

$$
u^{\varepsilon} \leq\left(\frac{1+\eta}{2}\right)^{j} \text { in } B_{r_{0} / 2^{j}} \times\left[T_{j}(\varepsilon), \infty\right) .
$$

Since (2.22) yields that (2.23) holds for $j=1$, we assume that (2.23) is valid for some $j \in \mathbb{N}$, set

$$
w^{\varepsilon}:=\left(\frac{2}{1+\eta}\right)^{j} u^{\varepsilon}\left(\cdot, \cdot+T_{j}(\varepsilon)\right) \text { in } Q
$$

observe that $w^{\varepsilon}$ is a solution of (2.1), with $a^{\varepsilon}(\cdot, \cdot)$ replaced by $a^{\varepsilon}\left(\cdot, \cdot+T_{j}(\varepsilon)\right)$ and satisfies $w^{\varepsilon}(0, t) \leq 0$ for all $t \in[0, \infty)$ and $w^{\varepsilon} \leq 1$ in $B_{r_{0} / 2^{j}} \times[0, \infty)$.

Using Lemma 1 and Corollary 6 as before, with the same $m$ and $\tau_{0}$, but with $u^{\varepsilon}, r_{0}$ and $\tau_{1}$ replaced by $w^{\varepsilon}, r_{0} / 2^{j}$ and $\tau_{j+1}$ respectively, we obtain

$$
w^{\varepsilon} \leq \frac{1+\eta}{2} \text { in } B_{r_{0} / 2^{j+1}} \times\left(\tau_{0}+\tau_{j+1}(\varepsilon), \infty\right),
$$

which, after been rewritten as

$$
u^{\varepsilon} \leq\left(\frac{1+\eta}{2}\right)^{j+1} \text { in } B_{r_{0} / 2^{j+1}} \times\left[T_{j+1}(\varepsilon), \infty\right),
$$

yields the claim.

Finally, selecting $j \in \mathbb{N}$ so that

$$
\left(\frac{1+\eta}{2}\right)^{j} \leq \delta
$$

setting $\varepsilon_{0}=\varepsilon_{j}, \gamma=2^{-j}$ and $\tau(\varepsilon)=T_{j}(\varepsilon)$, and observing that, as $\varepsilon \rightarrow 0+, \tau(\varepsilon)=O(|\log \varepsilon|)$ we complete the proof.

We have by now completed all the technical steps needed for the next theorem, which is a nontrivial refinement of Proposition 17. It asserts that bounded solutions to (2.1), which are small on the positive time axis $l$ and a part of the parabolic boundary, are actually small in almost the whole domain after some time of order $|\log \varepsilon|$. This is the mathematical statement of what we called asymptotic constancy.

Theorem 9. Assume (1.3), (1.6), (1.7), (1.8), (1.9) and (2.2) and let $\{T(\varepsilon)\}_{\varepsilon \in(0,1)}$ be a collection of positive numbers. For each $\delta>0$ and $C_{0}>0$, there exist constants $\varepsilon_{0} \in(0,1)$ and $C>0$ such that, if, for $\varepsilon \in\left(0, \varepsilon_{0}\right], u^{\varepsilon} \in C^{2,1}(Q)$ is a solution of (2.1) satisfying

$$
u^{\varepsilon} \leq C_{0} \quad \text { in } \Omega \times[0, T(\varepsilon)) \text { and } u^{\varepsilon} \leq 0 \text { in }(\{0\} \cup \Pi) \times[0, T(\varepsilon)) \text {, }
$$

then

$$
u^{\varepsilon}(x, t) \leq \delta \quad \text { for all }(x, t) \in \Omega_{\delta}^{\Pi} \times(C|\log \varepsilon|, T(\varepsilon)) .
$$


Proof. Proposition 8 yields constants $\varepsilon_{1}, \gamma \in(0,1)$ and $C_{1}>0$ such that, for all $0<\varepsilon \leq \varepsilon_{1}$,

$$
u^{\varepsilon} \leq \frac{\delta}{2} \text { in } B_{\gamma r_{0}} \times\left[C_{1}|\log \varepsilon|, T(\varepsilon)\right) .
$$

Proposition 7 applied to $v^{\varepsilon}(x, t):=C_{0}^{-1}\left(u^{\varepsilon}\left(x, t+C_{1}|\log \varepsilon|\right)-\delta\right)$ instead $u^{\varepsilon}$ implies the existence of $T_{\delta}$ and $\varepsilon_{0} \in\left(0, \varepsilon_{1}\right)$ such that, for any $\varepsilon \in\left(0, \varepsilon_{0}\right)$,

$$
v^{\varepsilon} \leq \frac{\delta}{2 C_{0}} \text { in } \Omega_{\delta}^{\Pi} \times\left[T_{\delta}, T(\varepsilon)-C_{1}|\log \varepsilon|\right),
$$

which says that, for any $\varepsilon \in\left(0, \varepsilon_{0}\right)$,

$$
u^{\varepsilon} \leq \delta \text { in } \Omega_{\delta}^{\Pi} \times\left[T_{\delta}+C_{1}|\log \varepsilon|, T(\varepsilon)\right),
$$

and the proof is complete.

Next we use the last result to control the difference between values of $u^{\varepsilon}(\cdot, t)$ and $u^{\varepsilon}(0, t)$.

Theorem 10. Assume (1.3), (1.6), (1.7), (1.8), (1.9) and (2.2). For each $\delta>0$ and $C_{0}>0$ there exist constants $\varepsilon_{0} \in(0,1)$ and $C>0$ such that, if, for $\varepsilon \in\left(0, \varepsilon_{0}\right]$, $u^{\varepsilon}$ is a solution of (2.1) satisfying

$$
\left|u^{\varepsilon}\right| \leq C_{0} \quad \text { in } \Omega \times[0, \infty),
$$

then

$$
\left|u^{\varepsilon}(x, t)-u^{\varepsilon}(0, t)\right| \leq \delta \quad \text { for all }(x, t) \in \Omega_{\delta} \times[C|\log \varepsilon|, \infty) .
$$

Proof. We double the variables and define the function $v^{\varepsilon}: \Omega \times \Omega \times[0, \infty) \rightarrow \mathbb{R}$ by

$$
v^{\varepsilon}(x, y, t):=u^{\varepsilon}(x, t)-u^{\varepsilon}(y, t) .
$$

It is standard that $v^{\varepsilon}$ solves in $\Omega \times \Omega \times(0, \infty)$ the doubled equation

$$
\begin{aligned}
v_{t}^{\varepsilon} & =\varepsilon \operatorname{tr}\left[a^{\varepsilon}(x, t) D_{x}^{2} v^{\varepsilon}\right]+\varepsilon \operatorname{tr}\left[a^{\varepsilon}(y, t) D_{y}^{2} v^{\varepsilon}\right]+b(x) \cdot D_{x} v^{\varepsilon}+b(y) \cdot D_{y} v^{\varepsilon} \\
& =\varepsilon \operatorname{tr}\left[A^{\varepsilon}(x, y, t) D^{2} v^{\varepsilon}\right]+B(x, y) \cdot D v^{\varepsilon},
\end{aligned}
$$

where

$$
B(x, y):=(b(x), b(y)) \quad \text { and } \quad A^{\varepsilon}(x, y, t):=\left(\begin{array}{cc}
a^{\varepsilon}(x, t) & 0 \\
0 & a^{\varepsilon}(y, t)
\end{array}\right) .
$$

The conclusion follows if we apply Theorem 9, with $\Pi=\emptyset$, to $\pm v^{\varepsilon}$, since $v^{\varepsilon}(0,0, t)=0$ for all $t \geq 0$ and $\left|v^{\varepsilon}\right| \leq 2 C_{0}$ in $\Omega \times \Omega \times[0, \infty)$.

The only issue is that the boundary of $\Omega \times \Omega$ does not have the $C^{1}$ - regularity required for the theorem.

To overcome this difficulty, we only need to approximate $\Omega \times \Omega$ by smaller $C^{1}$-domains, that is, for fixed $\delta>0$, we choose a $C^{1}$-domain $W \subset \mathbb{R}^{2 n}$ so that

$$
\Omega_{\delta} \times \Omega_{\delta} \subset W_{\delta / 2} \subset W \subset \Omega \times \Omega,
$$

where $W_{\delta / 2}:=\{(x, y) \in W: \operatorname{dist}((x, y), \partial W)>\delta / 2\}$, and

$$
B(x, y) \cdot N(x, y)<0 \quad \text { for all }(x, y) \in \partial W,
$$

where $N(x, y)$ denotes the outward unit normal vector at $(x, y) \in \partial W$. 


\section{QUASI-POTENTIALS}

We establish here an important continuity property under perturbations for the minimum and the arg min map of the quasi-potentials we introduced earlier in the introduction.

We begin with some notation and the introduction of several auxiliary quantities needed to define the perturbations. To this end, we fix $\beta_{0} \in I_{g}$, define $H_{0} \in C\left(\bar{\Omega} \times \mathbb{R}^{n}\right)$ by

$$
H_{0}(x, p)=a\left(x, \beta_{0}\right) p \cdot p+b(x) \cdot p,
$$

choose some $\delta_{0}>0$, and, for $\delta \in\left(0, \delta_{0}\right)$,

$$
\theta(\delta):=\max \left\{\left|\left(a(x, c)-a\left(x, \beta_{0}\right)\right) \xi \cdot \xi\right|: x \in \bar{\Omega}, \xi \in \mathbb{R}^{n},|\xi| \leq 1, c \in\left[\beta_{0}-\delta, \beta_{0}+\delta\right]\right\} .
$$

The continuity of $a(x, c)$ (recall (1.5) ) yields $\lim _{\delta \rightarrow 0} \theta(\delta)=0$, and, hence, selecting $\delta_{0}>0$ sufficiently small, we assume henceforth that

$$
\theta(\delta) \leq \theta_{0} / 2 \quad \text { for all } \delta \in\left(0, \delta_{0}\right) .
$$

We define $a_{\delta}^{ \pm} \in C\left(\bar{\Omega}, \mathbb{S}^{n}\right)$ and $H_{\delta}^{ \pm} \in C\left(\bar{\Omega} \times \mathbb{R}^{n}\right)$ respectively by

$$
a_{\delta}^{ \pm}(x):=a\left(x, \beta_{0}\right) \pm \theta(\delta) I \text { and } H_{\delta}^{ \pm}(x, p):=a_{\delta}^{ \pm}(x) p \cdot p+b(x) \cdot p,
$$

and note that, for all $(x, c) \in \bar{\Omega} \times\left[\beta_{0}-\delta, \beta_{0}+\delta\right]$,

$$
\left(\theta_{0} / 2\right) I \leq a_{\delta}^{-}(x) \leq a(x, c) \leq a_{\delta}^{+}(x) \leq\left(\theta_{0}^{-1}+\theta_{0} / 2\right) I .
$$

We choose $\chi_{\delta} \in C\left(\mathbb{R}^{n} ;[0,1]\right)$ such that

$$
\chi_{\delta}=1 \quad \text { in } \quad x \in \Omega_{\delta} \quad \text { and } \quad \chi_{\delta}=0 \quad \text { in } \quad \mathbb{R}^{n} \backslash \Omega_{\delta / 2},
$$

and define $\mathcal{H}_{\delta}^{ \pm} \in C\left(\bar{\Omega} \times \mathbb{R}^{n}\right)$ by

$$
\begin{aligned}
& \mathcal{H}_{\delta}^{+}(x, p)=\chi_{\delta}(x) H_{\delta}^{+}(x, p)+\left(1-\chi_{\delta}(x)\right)\left(\theta_{0}^{-1}|p|^{2}+b(x) \cdot p\right), \\
& \mathcal{H}_{\delta}^{-}(x, p)=\chi_{\delta}(x) H_{\delta}^{-}(x, p)+\left(1-\chi_{\delta}(x)\right)\left(\theta_{0}|p|^{2}+b(x) \cdot p\right),
\end{aligned}
$$

and note that, for all $(x, c) \in \Omega_{\delta / 2} \times\left[\beta_{0}-\delta, \beta_{0}+\delta\right] \cup\left(\Omega \backslash \Omega_{\delta / 2}\right) \times \mathbb{R}$ and $p \in \mathbb{R}^{n}$,

$$
\mathcal{H}_{\delta}^{-}(x, p) \leq a(x, c) p \cdot p+b(x) \cdot p \leq \mathcal{H}_{\delta}^{+}(x, p) .
$$

We also have

$$
\mathcal{H}_{\delta}^{ \pm}(x, p)=H_{\delta}^{ \pm}(x, p) \text { for all }(x, p) \in \Omega_{\delta} \times \mathbb{R}^{n},
$$

while, for all $(x, p) \in\left(\bar{\Omega} \backslash \Omega_{\delta / 2}\right) \times \mathbb{R}^{n}$,

$$
\mathcal{H}_{\delta}^{+}(x, p)=\theta_{0}^{-1}|p|^{2}+b(x) \cdot p \quad \text { and } \quad \mathcal{H}_{\delta}^{-}(x, p)=\theta_{0}|p|^{2}+b(x) \cdot p .
$$

If we set

$$
\alpha_{\delta}^{+}(x)=\chi_{\delta}(x) a_{\delta}^{+}(x)+\left(1-\chi_{\delta}(x)\right) \theta_{0}^{-1} I \quad \text { and } \quad \alpha_{\delta}^{-}(x)=\chi_{\delta}(x) a_{\delta}^{-}(x)+\left(1-\chi_{\delta}(x)\right) \theta_{0} I,
$$

then, for all $(x, p) \in \bar{\Omega} \times \mathbb{R}^{n}$,

$$
\mathcal{H}_{\delta}^{ \pm}(x, p)=\alpha_{\delta}^{ \pm}(x) p \cdot p+b(x) \cdot p .
$$

Let $V_{0}$ and $V_{\delta}^{ \pm}$be respectively the maximal subsolutions of

$$
\left\{\begin{array}{l}
H_{0}(x, D u)=0 \text { in } \Omega, \\
u(0)=0
\end{array}\right.
$$


and

$$
\left\{\begin{array}{l}
\mathcal{H}_{\delta}^{ \pm}(x, D u)=0 \quad \text { in } \Omega, \\
u(0)=0 .
\end{array}\right.
$$

We note by [11, Corollary 5] that $V_{\delta}^{ \pm}(x)>0$ and $V_{0}(x)>0$ for all $x \in \bar{\Omega} \backslash\{0\}$. Since $\mathcal{H}_{\delta}^{-} \leq H_{0} \leq \mathcal{H}_{\delta}^{+}$on $\Omega \times \mathbb{R}^{n}$, it is clear that

$$
V_{\delta}^{+} \leq V_{0} \leq V_{\delta}^{-} \text {on } \bar{\Omega} \text {. }
$$

We set

$$
M_{0}:=\min _{\partial \Omega} V_{0}, \quad \Gamma_{0}:=\arg \min \left(V_{0} \mid \partial \Omega\right), \quad M_{\delta}^{ \pm}:=\min _{\partial \Omega} V_{\delta}^{ \pm}, \quad \Gamma_{\delta}^{ \pm}:=\arg \min \left(V_{\delta}^{ \pm} \mid \partial \Omega\right),
$$

and note that

$$
M_{\delta}^{+} \leq M_{0} \leq M_{\delta}^{-} .
$$

The following result is about the continuity of $M_{\delta}^{ \pm}$and $\Gamma_{\delta}^{ \pm}$with respect to $\delta$.

Proposition 11. Assume (1.3), (1.5), (1.6), (1.7) and (1.8). Then

$$
\lim _{\delta \rightarrow 0+} M_{\delta}^{+}=\lim _{\delta \rightarrow 0+} M_{\delta}^{-}=M_{0}
$$

and

$$
\limsup _{\delta \rightarrow 0+} \Gamma_{\delta}^{+} \cup \limsup _{\delta \rightarrow 0+} \Gamma_{\delta}^{-} \subset \Gamma_{0} .
$$

The set limit in (3.5) is understood in the sense of Kuratowski, that is, for a given $\left\{\Gamma_{\delta}\right\}_{\delta \in\left(0, \delta_{0}\right)} \subset \mathbb{R}^{n}$,

$$
\limsup _{\delta \rightarrow 0+} \Gamma_{\delta}:=\bigcap_{r \in\left(0, \delta_{0}\right)} \overline{\bigcup_{\delta \in(0, r)} \Gamma_{\delta}}=\left\{x \in \mathbb{R}^{n}: x=\lim _{k \rightarrow \infty} x_{k}, x_{k} \in \Gamma_{\delta_{k}}, \lim _{k \rightarrow \infty} \delta_{k}=0\right\} .
$$

Now we prove Proposition 11 .

Proof. The uniform in $x$ and $\delta$ coercivity of the Hamiltonians $\mathcal{H}_{\delta}^{ \pm}$, that is the fact that $\mathcal{H}_{\delta}^{ \pm}(x, p) \rightarrow \infty$ as $|p| \rightarrow \infty$ uniformly in $x$ and $\delta$, yields that the families $\left\{V_{\delta}^{ \pm}\right\}_{\delta \in\left(0, \delta_{0}\right)}$ are equi-Lipschitz continuous on $\bar{\Omega}$, and, since $V_{\delta}^{ \pm}(0)=0$, relatively compact in $C(\bar{\Omega})$.

To prove (3.4) and (3.5), it is enough to show that, if $\left\{\delta_{j}\right\}_{j \in \mathbb{N}} \subset\left(0, \delta_{0}\right)$ is such that both $\left\{V_{\delta_{j}}^{ \pm}\right\}_{j \in \mathbb{N}}$ converge in $C(\bar{\Omega})$ to some $V_{0}^{ \pm} \in C(\bar{\Omega})$, that is

$$
V_{0}^{ \pm}=\lim _{j \rightarrow \infty} V_{\delta_{j}}^{ \pm} \quad \text { uniformly on } \bar{\Omega},
$$

then

$$
M_{0}=\min _{\partial \Omega} V_{0}^{+}=\min _{\partial \Omega} V_{0}^{-}
$$

and

$$
\arg \min \left(V_{0} \mid \partial \Omega\right)=\arg \min \left(V_{0}^{+} \mid \partial \Omega\right)=\arg \min \left(V_{0}^{-} \mid \partial \Omega\right) .
$$

For notational convenience, we set

$$
M_{0}^{ \pm}:=\min _{\partial \Omega} V_{0}^{ \pm} \quad \text { and } \quad \Gamma_{0}^{ \pm}=\arg \min \left(V_{0}^{ \pm} \mid \partial \Omega\right) .
$$

It is well-known (see Lemma B.1 in the Appendix) that the $V_{\delta}^{ \pm}$'s satisfy in the viscosity sense

$$
\mathcal{H}_{\delta}^{ \pm}\left(x, D V_{\delta}^{ \pm}\right) \geq 0 \quad \text { on } \bar{\Omega} \quad \text { and } \quad \mathcal{H}_{\delta}^{ \pm}\left(x, D V_{\delta}^{ \pm}\right) \leq 0 \text { in } \Omega,
$$


that is, the $V_{\delta}^{ \pm}$'s are solutions of the state-constraints problems

$$
\mathcal{H}_{\delta}^{ \pm}\left(x, D V_{\delta}^{ \pm}\right)=0 \quad \text { in } \Omega .
$$

By the stability of viscosity properties, the $V_{0}^{ \pm}$'s satisfy

$$
H_{0}\left(x, D V_{0}^{ \pm}(x)\right) \leq 0 \quad \text { in } \Omega \quad \text { and } \quad H_{\theta_{0}}^{+}\left(x, D V_{0}^{+}(x)\right) \geq 0 \quad \text { on } \bar{\Omega},
$$

where

$$
H_{\theta_{0}}^{+}(x, p):= \begin{cases}H_{0}(x, p) & \text { for }(x, p) \in \Omega \times \mathbb{R}^{n}, \\ \theta_{0}^{-1}|p|^{2}+b(x) \cdot p & \text { for }(x, p) \in \partial \Omega \times \mathbb{R}^{n} .\end{cases}
$$

Here we used that

$$
\limsup _{\delta \rightarrow 0} * \mathcal{H}_{\delta}^{ \pm}(x, p)=\liminf _{\delta \rightarrow 0} \mathcal{H}_{\delta}^{ \pm}(x, p)=H_{0}(x, p) \quad \text { for all }(x, p) \in \Omega \times \mathbb{R}^{n},
$$

and

$$
\limsup _{\delta \rightarrow 0} \mathcal{H}_{\delta}^{+}(x, p)=H_{\theta_{0}}^{+}(x, p) \quad \text { for all }(x, p) \in \bar{\Omega} \times \mathbb{R}^{n} .
$$

The maximality of $V_{0}$ implies that $V_{0}^{-} \leq V_{0}$ on $\Omega$ and, since, in view of (3.3), $V_{0} \leq$ $V_{0}^{-}$in $\bar{\Omega}$, we have $V_{0}^{-}=V_{0}$, which, obviously gives

$$
M_{0}=M_{0}^{-} \quad \text { and } \quad \Gamma_{0}^{-}=\Gamma_{0} .
$$

The argument for $M_{0}^{+}$and $\Gamma_{0}^{+}$is slightly more complicated.

Since (3.3) yields $V_{0}^{+} \leq V_{0}$, it is immediate that

$$
M_{0}^{+} \leq M_{0}
$$

Next we show that

$$
\min \left\{V_{0}, M_{0}\right\} \leq V_{0}^{+} \text {in } \bar{\Omega},
$$

which, together the previous inequality, gives

$$
M_{0}^{+}=M_{0} \quad \text { and } \quad \Gamma_{0} \subset \Gamma_{0}^{+} .
$$

We proceed with the proof of (3.9) . Fix $l \in\left(0, M_{0}\right)$, choose $\gamma_{1} \in\left(0, \delta_{0}\right)$ so that

$$
V_{0}>l \text { on } \bar{\Omega} \backslash \Omega_{\gamma_{1}},
$$

fix $\mu \in(0,1)$ sufficiently close to 1 so that

$$
\mu V_{0}>l \text { on } \bar{\Omega} \backslash \Omega_{\gamma_{1}},
$$

and choose $\gamma_{2} \in\left(0, \gamma_{1}\right)$ so that

$$
\mu\left(a\left(x, \beta_{0}\right)+\theta(\delta) I\right) \leq a\left(x, \beta_{0}\right) \quad \text { for all } x \in \bar{\Omega} \text { and } \delta \in\left(0, \gamma_{2}\right) .
$$

Observe that, if $u_{\mu}(x):=\mu V_{0}(x)$, then, for all $\delta \in\left(0, \gamma_{2}\right)$,

$$
u_{\mu}>l \text { in } \bar{\Omega} \backslash \Omega_{\delta},
$$

and, for all $\delta \in\left(0, \gamma_{2}\right)$, in the viscosity sense,

$$
\begin{aligned}
H_{\delta}^{+}\left(x, D u_{\mu}\right) & =\mu\left(\mu\left(a\left(x, \beta_{0}\right)+\theta(\delta) I\right) D V_{0} \cdot D V_{0}+b(x) \cdot D V_{0}\right) \\
& \leq \mu\left(a\left(x, \beta_{0}\right) D V_{0} \cdot D V_{0}+b \cdot D V_{0}\right) \leq \mu H_{0}\left(x, D V_{0}\right) \leq 0 \quad \text { in } \Omega .
\end{aligned}
$$

Now set $u_{\mu}^{l}:=\min \left\{u_{\mu}, l\right\}$ and note that the convexity of $H_{\delta}^{+}(x, p)$ in $p$ yields that, if $\delta \in\left(0, \gamma_{2}\right)$, then

$$
\mathcal{H}_{\delta}^{+}\left(x, D u_{\mu}^{l}\right)=H_{\delta}^{+}\left(x, D u_{\mu}^{l}\right) \leq 0 \quad \text { in } \quad \Omega_{\delta} .
$$


Also, if $\delta \in\left(0, \gamma_{2}\right)$, then, since $u_{\mu}^{l}(x)=l$ in an open neighborhood $N_{\delta} \subset \Omega$ of $\Omega \backslash \Omega_{\delta}$,

$$
\mathcal{H}_{\delta}\left(x, D u_{\mu}^{l}\right)=0 \quad \text { in } N_{\delta} .
$$

Thus we deduce that, for any $\delta \in\left(0, \gamma_{2}\right), u_{\mu}^{l}$ is a subsolution of $\mathcal{H}_{\delta}^{+}\left(x, D u_{\mu}^{l}\right) \leq 0$ in $\Omega$, and, hence, $u_{\mu}^{l} \leq V_{\delta}^{+}$in $\bar{\Omega}$ by the maximality of $V_{\delta}^{+}$. Sending $\delta \rightarrow 0$, along the sequence $\left\{\delta_{j}\right\}, \mu \rightarrow 1$ and $l \rightarrow M_{0}$ in this order, we conclude that (3.9) holds.

Next we show that $\Gamma_{0}^{+} \subset \Gamma_{0}$. Let $z \in \Gamma_{0}^{+} \backslash \Gamma_{0}$ and observe that, since $V_{0}(z)>M_{0}$, there is an open, relatively to $\bar{\Omega}$, neighborhood $N_{z} \subset \bar{\Omega}$, such that $V_{0}>M_{0}$ in $N_{z}$, while (3.9) gives $V_{0}^{+} \geq M_{0}$ in $N_{z}$.

Let $\rho \in C^{1}\left(\mathbb{R}^{n}\right)$ be a defining function of $\Omega$, that is, $\Omega=\left\{x \in \mathbb{R}^{n}: \rho(x)<0\right\}$ and $|D \rho| \neq 0$ on $\partial \Omega$, and, in particular, $D \rho /|D \rho|=\nu$ on $\partial \Omega$.

For any $\varepsilon>0, x \mapsto V_{0}^{+}(x)-\varepsilon \rho(x)$ achieves a minimum at $z$ over $N_{z}$. Since $H_{\theta_{0}}^{+}\left(x, D V_{0}^{+}\right) \geq$ 0 on $\bar{\Omega}$, we have

$$
0 \leq H_{\theta_{0}}^{+}(z, \varepsilon D \rho(z))=\varepsilon\left(\varepsilon \theta_{0}^{-1}|D \rho(z)|^{2}+b(z) \cdot D \rho(z)\right),
$$

which is a contradiction, in view of the fact that the right hand side is negative if $\varepsilon$ is sufficiently small.

It follows that $\Gamma_{0}^{+} \backslash \Gamma_{0}=\emptyset$, that is, $\Gamma_{0}^{+} \subset \Gamma_{0}$, which, together with (3.10), proves the claim.

\section{BARRIER FUNCTIONS}

We adapt and modify here the main argument of building barrier functions of [1] to obtain information on the behavior of the solutions $u^{\varepsilon}$ of (2.1) along the positive time axis $l$, that is on $u^{\varepsilon}(0, t)$, for a sufficiently long time interval $[0, T(\varepsilon))$, under the assumption that the matrices $a^{\varepsilon} \in C\left(\bar{Q}_{T(\varepsilon)}\right)$ are bounded by $\alpha \in C\left(\bar{Q}_{T(\varepsilon)}\right)$ from above or from below.

Recall that, for any $\alpha \in C\left(\bar{\Omega}, \mathbb{S}^{n}\left(\theta_{0}\right)\right), H_{\alpha} \in C\left(\bar{\Omega} \times \mathbb{R}^{n}\right)$ is the Hamiltonian given by $H_{\alpha}(x, p)=\alpha(x) p \cdot p+b(x) \cdot p, V_{\alpha} \in \operatorname{Lip}(\bar{\Omega})$ is the quasi-potential corresponding to $(\alpha, b)$, and $M_{\alpha}=\min _{\partial \Omega} V_{\alpha}$, and set

$$
\Sigma_{\alpha}:=\left\{x \in \bar{\Omega}: V_{\alpha}(x) \leq M_{\alpha}\right\}, \quad \Gamma_{\alpha}:=\Sigma_{\alpha} \cap \partial \Omega,
$$

and, for any $m>0$,

$$
\Sigma_{\alpha}^{m}:=\left\{x \in \bar{\Omega}: V_{\alpha}(x) \leq m\right\} .
$$

We consider again (2.1) for a family of $a^{\varepsilon} \in C\left(\bar{Q}, \mathbb{S}^{n}\left(\theta_{0}\right)\right)$ and present two results, one stated in the form of an upper bound and the other in the form of a lower bound. The upper bound is valid up to $\lambda$ smaller than $M_{\alpha}$ in the logarithmic time scale, and the lower bound is valid up to $\infty$, provided $u^{\varepsilon}$, on the boundary portion $\Gamma_{\alpha} \times[0, T(\varepsilon)$ ), is larger than a specified lower bound.

We begin with the former, which corresponds to [11, Theorem 1 (i)] in its nature. The latter is related to [11, Theorem 1(ii)].

Proposition 12. Assume (1.10) and fix $\alpha \in C\left(\bar{\Omega}, \mathbb{S}^{n}\left(\theta_{0}\right)\right), T(\varepsilon) \in(0, \infty], C_{0}>0$ and $m \in$ $\left(0, M_{\alpha}\right)$. If, for $a^{\varepsilon} \in C\left(\bar{Q}_{T(\varepsilon)} ; \mathbb{S}^{n}\left(\theta_{0}\right)\right)$ such that $a^{\varepsilon} \leq \alpha$ in $Q_{T(\varepsilon)}, u^{\varepsilon} \in C\left(\bar{Q}_{T(\varepsilon)}\right) \cap C^{2,1}\left(Q_{T(\varepsilon)}\right)$ is a subsolution of (2.1) in $Q_{T(\varepsilon)}$ such that

$$
u^{\varepsilon}(\cdot, 0) \leq 0 \text { in } \Sigma_{\alpha}^{m} \text { and } u^{\varepsilon} \leq C_{0} \text { in } Q_{T(\varepsilon)} \text {, }
$$


then, for any $\delta>0$, there exists $\varepsilon_{0} \in(0,1)$ such that, if $\varepsilon \in\left(0, \varepsilon_{0}\right)$, then

$$
u^{\varepsilon}(0, t) \leq \delta \quad \text { for all } t \in[0, \exp ((m-\delta) / \varepsilon) \wedge T(\varepsilon)) .
$$

The lower bound is stated next.

Proposition 13. Assume (1.10), fix $\alpha \in C\left(\bar{\Omega}, \mathbb{S}^{n}\left(\theta_{0}\right)\right), T(\varepsilon) \in(0, \infty], C_{0}>0$ and $m>$ $M_{\alpha}$. If, for $a^{\varepsilon} \in C\left(\bar{Q}_{T(\varepsilon)} ; \mathbb{S}^{n}\left(\theta_{0}\right)\right)$ such that $a^{\varepsilon} \geq \alpha$ in $Q_{T(\varepsilon)}, u^{\varepsilon} \in C\left(\bar{Q}_{T(\varepsilon)}\right) \cap C^{2,1}\left(Q_{T(\varepsilon)}\right)$ is a supersolution of (2.1) in $Q_{T(\varepsilon)}$ such that

$$
u^{\varepsilon}(\cdot, 0) \geq 0 \quad \text { in } \quad \Sigma_{\alpha}^{m}, \quad u^{\varepsilon} \geq 0 \quad \text { in } \quad\left(\Sigma_{\alpha}^{m} \cap \partial \Omega\right) \times(0, T(\varepsilon)) \text { and } u^{\varepsilon} \geq-C_{0} \quad \text { in } Q_{T(\varepsilon)},
$$

then, for any $\delta>0$, there exists $\varepsilon_{0} \in(0,1)$ such that, if $\varepsilon \in\left(0, \varepsilon_{0}\right)$, then

$$
u^{\varepsilon}(0, t) \geq-\delta \quad \text { for all } t \in[0, T(\varepsilon)) .
$$

The proofs of Propositions 12 and 13 use the next two lemmata; for their proof we refer to 11 .

Lemma 3. Assume (1.10) and fix $\alpha \in C\left(\bar{\Omega}, \mathbb{S}^{n}\left(\theta_{0}\right)\right)$. For any $r \in\left(0, r_{0}\right)$, there exist $v_{r} \in C^{2}(\bar{\Omega})$ and $\eta \in(0,1)$ such that

$$
\left\{\begin{array}{l}
H_{\alpha}\left(x, D v_{r}\right) \leq-\eta \quad \text { in } \Omega \backslash B_{r}, \\
H_{\alpha}\left(x, D v_{r}\right) \leq 1 \text { in } B_{r}, \\
\left\|v_{r}-V_{\alpha}\right\|_{L^{\infty}(\Omega)}<r .
\end{array}\right.
$$

Lemma 4. Assume (1.10) and fix $\alpha \in C\left(\bar{\Omega}, \mathbb{S}^{n}\left(\theta_{0}\right)\right)$. For each $m>M_{\alpha}$, there exists $w_{m} \in \operatorname{Lip}(\bar{\Omega})$ and $\eta>0$ such that

$$
0<\min _{\bar{\Omega}} w_{m} \leq \max _{\bar{\Omega}} w_{m}<m,
$$

and, in the viscosity supersolution sense,

$$
H_{\alpha}\left(x,-D w_{m}\right) \geq \eta \quad \text { in } \Omega \text { and } D^{2} w_{m}(x) \leq \eta^{-1} I \text { in } \Omega .
$$

We continue with the proof of Proposition 12 which parallels that of [11, Theorem 8].

Proof of Proposition [12. For $r \in\left(0, r_{0}\right)$ to be fixed below, let $v=v_{r} \in C^{2}(\bar{\Omega})$ (for notational simplicity we omit the subscript $r$ in what follows) and $\eta>0$ be given by Lemma 3 , set, for $x \in \bar{\Omega}$,

$$
w^{\varepsilon}(x):=\exp \left(\frac{v(x)-m+2 r}{\varepsilon}\right)
$$

compute, for any $(x, t) \in Q$,

$$
\begin{aligned}
\varepsilon \operatorname{tr}\left[a^{\varepsilon}(x, t)\right. & \left.D^{2} w^{\varepsilon}\right]+b(x) \cdot D w^{\varepsilon} \\
& =\frac{w^{\varepsilon}}{\varepsilon}\left(a^{\varepsilon}(x, t) D v \cdot D v+b \cdot D v+\varepsilon \operatorname{tr}\left[a^{\varepsilon}(x, t) D^{2} v\right]\right) \\
& \leq \frac{w^{\varepsilon}}{\varepsilon}\left(\alpha(x) D v \cdot D v+b(x) \cdot D v+\varepsilon \operatorname{tr}\left[a^{\varepsilon}(x, t) D^{2} v\right]\right) \\
& \leq \frac{w^{\varepsilon}}{\varepsilon}\left(H_{\alpha}(x, D v)+\varepsilon \operatorname{tr}\left[a^{\varepsilon}(x, t) D^{2} v\right]\right) .
\end{aligned}
$$

and choose $\varepsilon_{0} \in(0,1)$ so that

$$
\varepsilon_{0}\left(\operatorname{tr} a^{\varepsilon}(x, t) D^{2} v\right)_{+} \leq \min \{\eta, r, 1\}
$$

note that $\varepsilon_{0}$ can be chosen so as to depend on $a^{\varepsilon}$ only through $\theta_{0}$. 
We assume henceforth that $\varepsilon \in\left(0, \varepsilon_{0}\right)$ and observe that, from the computation above, we get

$$
\varepsilon \operatorname{tr}\left[a^{\varepsilon}(x, t) D^{2} w^{\varepsilon}\right]+b(x) \cdot D w^{\varepsilon} \leq\left\{\begin{array}{l}
0 \text { for all }(x, t) \in \Omega \backslash B_{r} \times(0, \infty), \\
\frac{2}{\varepsilon} w^{\varepsilon} \text { for all }(x, t) \in B_{r} \times(0, \infty) .
\end{array}\right.
$$

Let $C_{0}>0$ be a Lipschitz bound of $b$, and note that, if $H_{\alpha}(x, p) \leq 0$, then $|p| \leq C_{0} \theta_{0}^{-1}$, which implies that $V_{\alpha}(x) \leq C_{0}|x|^{2} /\left(2 \theta_{0}\right) \leq C_{0} r^{2} /\left(2 \theta_{0}\right)$ for all $x \in B_{r}$. We may thus assume by replacing, if needed, $r>0$ by a smaller number that $V_{\alpha} \leq r$ in $B_{r}$. Accordingly we have

$$
v-m+2 r \leq V_{\alpha}-m+3 r \leq-m+4 r \text { in } B_{r},
$$

and

$$
w^{\varepsilon} \leq \exp \left(\frac{-m+4 r}{\varepsilon}\right) \text { in } B_{r}
$$

Observe also that

$$
v-m+2 r>V_{\alpha}-m+r \geq r \text { in } \bar{\Omega} \backslash \Sigma_{\alpha}^{m},
$$

and

$$
w^{\varepsilon}>\exp \left(\frac{r}{\varepsilon}\right) \quad \text { in } \bar{\Omega} \backslash \Sigma_{\alpha}^{m} .
$$

Next set $d_{\varepsilon}=\frac{2}{\varepsilon} \exp \left(\frac{-m+4 r}{\varepsilon}\right)$ and

$$
z^{\varepsilon}(x, t)=w^{\varepsilon}(x)+d_{\varepsilon} t \quad \text { for } \quad(x, t) \in \bar{\Omega} \times[0, \infty) .
$$

It is immediate from (4.4) and (4.5) that

$$
z_{t}^{\varepsilon} \geq \varepsilon \operatorname{tr}\left[a^{\varepsilon} D^{2} z^{\varepsilon}\right]+b \cdot D z^{\varepsilon} \quad \text { in } Q .
$$

We choose $C_{1}>0$ so that, for all $\varepsilon \in(0,1)$,

$$
u^{\varepsilon} \leq C_{1} \text { on } \bar{Q},
$$

and by replacing, if necessary, $\varepsilon_{0}>0$ by a smaller number we may assume that, for all $\varepsilon \in\left(0, \varepsilon_{0}\right)$,

It follows from (4.6) that

$$
C_{1}<\exp \left(\frac{r}{\varepsilon}\right)
$$

$$
z^{\varepsilon} \geq w^{\varepsilon} \geq \exp \left(\frac{r}{\varepsilon}\right)>C_{1} \geq u^{\varepsilon} \text { on }\left(\bar{\Omega} \backslash \Sigma_{\alpha}^{m}\right) \times[0, \infty) ;
$$

note that, since $m<M_{\alpha}$, we have $\partial \Omega \subset \bar{\Omega} \backslash \Sigma_{\alpha}^{m}$.

On the other hand, for any $x \in \Sigma_{\alpha}^{m}$, we have

$$
z^{\varepsilon}(x, 0)=w^{\varepsilon}(x)>0 \geq u^{\varepsilon}(x, 0),
$$

and, hence,

$$
u^{\varepsilon} \leq z^{\varepsilon} \text { on } \partial_{\mathrm{p}} Q
$$

We find from the above, (4.7) and the comparison principle that

$$
u^{\varepsilon} \leq z^{\varepsilon} \text { on } \bar{Q},
$$

and, in particular, for any $t \in[0, \exp ((m-5 r) / \varepsilon)]$,

$$
u^{\varepsilon}(0, t) \leq z^{\varepsilon}(0, t) \leq w^{\varepsilon}(0)+\frac{2}{\varepsilon} \exp \left(\frac{-r}{\varepsilon}\right) \leq \exp \left(\frac{-m+3 r}{\varepsilon}\right)+\frac{2}{\varepsilon} \exp \left(\frac{-r}{\varepsilon}\right) .
$$


It is now clear that, for a given $\delta>0$, we may choose $r>0$ and $\varepsilon_{0} \in(0,1)$ so that if $\varepsilon \in\left(0, \varepsilon_{0}\right)$, then

$$
u^{\varepsilon}(0, t) \leq \exp \left(\frac{-m+3 r}{\varepsilon}\right)+\frac{2}{\varepsilon} \exp \left(\frac{-r}{\varepsilon}\right)<\delta \quad \text { for all } t \in[0, \exp ((m-\delta) / \varepsilon)] .
$$

We continue with

Proof of Proposition 13. We fix $r \in\left(0, r_{0}\right)$ small enough so that, as in the previous proof, $V_{\alpha} \leq r$ in $B_{r}$, and $m-5 r>M_{\alpha}$. In view of Lemmata 3 and 4 , we may choose $v \in C^{2}(\bar{\Omega})$, $w \in \operatorname{Lip}(\bar{\Omega})$ and $\eta>0$ so that, in addition to (4.1), $0<\min _{\bar{\Omega}} w<\max _{\bar{\Omega}} w<m-5 r$, and, in the viscosity supersolution sense,

$$
H_{\alpha}(x,-D w) \geq \eta \quad \text { and } \quad D^{2} w \leq \eta^{-1} I \quad \text { in } \Omega .
$$

Setting $u=-w, \rho^{-}=\min _{\bar{\Omega}} w$ and $\rho^{+}=\max _{\bar{\Omega}} w$, we get that $\rho^{+}<m-5 r, 0>-\rho^{-} \geq$ $u \geq-\rho^{+}$on $\bar{\Omega}$ and, in the viscosity subsolution sense,

$$
H_{\alpha}(x, D u) \geq \eta \quad \text { and } \quad D^{2} u \geq-\eta^{-1} I \quad \text { in } \Omega .
$$

For $\varepsilon \in(0,1)$, we set

$$
z^{\varepsilon}=-\exp \left(\frac{v-m+2 r}{\varepsilon}\right)+\exp \left(\frac{u}{\varepsilon}\right)-\exp \left(\frac{-\rho^{-}}{\varepsilon}\right)
$$

and find that, in the viscosity subsolution sense,

$$
\begin{aligned}
\varepsilon \operatorname{tr}\left[a^{\varepsilon} D^{2} z^{\varepsilon}\right]+b \cdot D z^{\varepsilon} \geq & -\frac{1}{\varepsilon} \exp \left(\frac{v-m+2 r}{\varepsilon}\right)\left(H_{\alpha}(x, D v)+\varepsilon \operatorname{tr}\left[a^{\varepsilon} D^{2} v\right]\right) \\
& +\frac{1}{\varepsilon} \exp \left(\frac{u}{\varepsilon}\right)\left(H_{\alpha}(x, D u)+\varepsilon \operatorname{tr}\left[a^{\varepsilon} D^{2} u\right]\right) \quad \text { in } Q .
\end{aligned}
$$

Let $\varepsilon_{0} \in(0,1)$ be a constant to be specified later and assume henceforth that $\varepsilon \in\left(0, \varepsilon_{0}\right)$. Observing that in the viscosity subsolution sense,

$$
\varepsilon \operatorname{tr}\left[a^{\varepsilon} D^{2} u\right] \geq-\eta^{-1} \operatorname{tr} a^{\varepsilon} \geq-n\left(\theta_{0} \eta\right)^{-1} \quad \text { in } Q,
$$

and

$$
\operatorname{tr}\left[a^{\varepsilon} D^{2} v\right] \leq\left\|D^{2} v\right\|_{L^{\infty}(\Omega)} \operatorname{tr} a^{\varepsilon} \leq n \theta_{0}^{-1}\left\|D^{2} v\right\|_{L^{\infty}(\Omega)} \quad \text { in } Q
$$

and, if for $x \in \bar{\Omega}$,

$$
\begin{aligned}
f(x):= & -\frac{1}{\varepsilon} \exp \left(\frac{v(x)-m+2 r}{\varepsilon}\right)\left(H_{\alpha}(x, D v(x))+\varepsilon n \theta_{0}^{-1}\left\|D^{2} v\right\|_{L^{\infty}(\Omega)}\right) \\
& +\frac{1}{\varepsilon} \exp \left(\frac{u(x)}{\varepsilon}\right)\left(\eta-\varepsilon n\left(\eta \theta_{0}\right)^{-1}\right),
\end{aligned}
$$

we obtain, in the viscosity subsolution sense,

$$
\varepsilon \operatorname{tr}\left[a^{\varepsilon} D^{2} z^{\varepsilon}\right]+b \cdot D z^{\varepsilon} \geq f \text { in } Q .
$$

Choosing $\varepsilon_{0} \in(0,1)$ so that

$$
\varepsilon_{0} n \theta_{0}^{-1}\left\|D^{2} v\right\|_{L^{\infty}(\Omega)} \leq \min \{\eta, 1\} \text { and } \varepsilon_{0} n\left(\eta \theta_{0}\right)^{-1} \leq \frac{\eta}{2},
$$

we get

$$
\eta-\varepsilon n\left(\eta \theta_{0}\right)^{-1} \geq \frac{\eta}{2} \quad \text { and } \quad H_{\alpha}(x, D v)+\varepsilon n \theta_{0}^{-1}\left\|D^{2} v\right\|_{L^{\infty}(\Omega)} \leq \begin{cases}0 & \text { for all } x \in \Omega \backslash B_{r} \\ 2 & \text { for all } x \in B_{r}\end{cases}
$$


and, accordingly,

$$
f \geq \begin{cases}0 & \text { in } \Omega \backslash B_{r}, \\ -\frac{2}{\varepsilon} \exp \left(\frac{-m+4 r}{\varepsilon}\right)+\frac{\eta}{2 \varepsilon} \exp \left(\frac{-\rho^{+}}{\varepsilon}\right) & \text { in } B_{r} .\end{cases}
$$

Since $\rho^{+}<m-5 r$, we have

$$
\begin{aligned}
-2 \exp \left(\frac{-m+4 r}{\varepsilon}\right)+\frac{\eta}{2} \exp \left(\frac{-\rho^{+}}{\varepsilon}\right) & \geq-2 \exp \left(\frac{-\rho^{+}-r}{\varepsilon}\right)+\frac{\eta}{2} \exp \left(\frac{-\rho^{+}}{\varepsilon}\right) \\
& =\exp \left(\frac{-\rho^{+}}{\varepsilon}\right)\left(-2 \exp \left(\frac{-r}{\varepsilon}\right)+\frac{\eta}{2}\right) .
\end{aligned}
$$

We may assume by replacing $\varepsilon_{0} \in(0,1)$ by a smaller number that

$$
2 \exp \left(\frac{-r}{\varepsilon_{0}}\right) \leq \frac{\eta}{2}
$$

and, therefore,

$$
-2 \exp \left(\frac{-m+4 r}{\varepsilon}\right)+\frac{\eta}{2} \exp \left(\frac{-\rho^{+}}{\varepsilon}\right) \geq 0,
$$

which ensures that $f \geq 0$ in $\Omega$, and, hence, $z^{\varepsilon}$, as a function of $(x, t) \in Q$, is a subsolution of (2.1).

Next observe that

$$
z^{\varepsilon}<0 \text { on } \bar{\Omega}
$$

and, if $V_{\alpha}(x)>m$,

$$
z^{\varepsilon}(x) \leq-\exp \left(\frac{V_{\alpha}(x)-m+r}{\varepsilon}\right) \leq-\exp \left(\frac{r}{\varepsilon}\right) .
$$

Fix $C_{1}>0$ so that, for $\varepsilon \in(0,1), u^{\varepsilon} \geq-C_{1}$ on $\bar{Q}$, and, assume henceforth that $\varepsilon_{0} \in(0,1)$ is small enough so that

$$
\exp \left(\frac{r}{\varepsilon_{0}}\right) \geq C_{1} .
$$

Consequently, we have

$$
z^{\varepsilon} \leq \begin{cases}-\exp \left(\frac{r}{\varepsilon}\right) \leq-C_{1} \leq u^{\varepsilon} & \text { in }\left(\bar{\Omega} \backslash \Sigma_{\alpha}^{m}\right) \times[0, T(\varepsilon)), \\ 0 \leq u^{\varepsilon}(\cdot, 0) & \text { in } \Sigma_{\alpha}^{m}, \\ 0 \leq u^{\varepsilon} & \text { in }\left(\Sigma_{\alpha}^{m} \cap \partial \Omega\right) \times(0, T(\varepsilon)),\end{cases}
$$

that is

$$
z^{\varepsilon} \leq u^{\varepsilon} \quad \text { on } \quad \partial_{\mathrm{p}} Q_{T(\varepsilon)},
$$

and, hence, by the comparison principle,

$$
z^{\varepsilon} \leq u^{\varepsilon} \text { on } \bar{Q}_{T(\varepsilon)} .
$$

Finally, we note that

$$
\begin{aligned}
z^{\varepsilon}(0) & =-\exp \left(\frac{v(0)-m+2 r}{\varepsilon}\right)-\exp \left(\frac{-\rho^{-}}{\varepsilon}\right) \\
& \geq-\exp \left(\frac{-m+4 r}{\varepsilon}\right)-\exp \left(\frac{-\rho^{-}}{\varepsilon}\right) \rightarrow 0 \quad \text { as } \varepsilon \rightarrow 0,
\end{aligned}
$$

which completes the proof. 
The following corollary is a variant of [11, Theorem 4.1]. Since its proof is similar to the one of Proposition 13 above, here we present only an outline.

Corollary 14. Assume (1.10), fix $\alpha \in C\left(\bar{\Omega}, \mathbb{S}^{n}\left(\theta_{0}\right)\right), T(\varepsilon) \in(0, \infty], C>0$ and $m>M_{\alpha}$. If, for $a^{\varepsilon} \in C\left(\bar{Q}_{T(\varepsilon)} ; \mathbb{S}^{n}\left(\theta_{0}\right)\right)$ such that $a^{\varepsilon} \geq \alpha$ in $Q_{T(\varepsilon)}, u^{\varepsilon} \in C\left(\bar{Q}_{T(\varepsilon)}\right) \cap C^{2,1}\left(Q_{T(\varepsilon)}\right)$ is a supersolution of (2.1) in $Q_{T(\varepsilon)}$ such that

$$
u^{\varepsilon} \geq 0 \quad \text { in }\left(\Sigma_{\alpha}^{m} \cap \partial \Omega\right) \times(0, T(\varepsilon)) \text { and } u^{\varepsilon} \geq-C \text { in } Q_{T(\varepsilon)},
$$

then, for any $\delta>0$, there exists $\varepsilon_{0} \in(0,1)$ such that, if $\varepsilon \in\left(0, \varepsilon_{0}\right)$, then

$$
u^{\varepsilon}(0, t) \geq-\delta \quad \text { for all } t \in[\exp (m / \varepsilon), T(\varepsilon)) .
$$

Outline of proof. Let $r \in\left(0, r_{0}\right), \eta>0, \rho^{ \pm}, v \in C^{2}(\bar{\Omega})$, and $w, u, z^{\varepsilon}, f \in \operatorname{Lip}(\bar{\Omega})$ be the same as in the proof of Proposition 13. According to (4.8), we have in the viscosity subsolution sense,

$$
\varepsilon \operatorname{tr}\left[a^{\varepsilon} D^{2} z^{\varepsilon}\right]+b \cdot D z^{\varepsilon} \geq f \quad \text { in } Q .
$$

Choosing $\varepsilon_{0} \in(0,1)$ so that

$$
\varepsilon_{0} n \theta_{0}^{-1}\left\|D^{2} v\right\|_{L^{\infty}(\Omega)} \leq \min \{\eta, 1\}, \quad \varepsilon_{0} n(\eta \theta)^{-1} \leq \frac{\eta}{2} \quad \text { and } \quad 2 \exp \left(\frac{-r}{\varepsilon_{0}}\right) \leq \frac{\eta}{4}
$$

and noting that, in $\Omega \backslash B_{r}$ and $\varepsilon \in\left(0, \varepsilon_{0}\right)$,

$$
f \geq \frac{1}{\varepsilon} \exp \left(\frac{u}{\varepsilon}\right)\left(\eta-\varepsilon n(\eta \theta)^{-1}\right) \geq \frac{\eta}{2} \exp \left(\frac{-\rho^{+}}{\varepsilon}\right),
$$

we compute, as in the proof of Proposition 13, to get, for any $\varepsilon \in\left(0, \varepsilon_{0}\right)$, that

$$
f \geq \exp \left(\frac{-\rho^{+}}{\varepsilon}\right)\left(-2 \exp \left(\frac{-r}{\varepsilon}\right)+\frac{\eta}{2}\right) \geq \frac{\eta}{4} \exp \left(-\frac{\rho^{+}}{\varepsilon}\right) \text { in } \Omega .
$$

Now, we fix $\gamma \in(0, \eta]$, set, for $(x, t) \in \bar{Q}$ and $\varepsilon \in\left(0, \varepsilon_{0}\right)$,

$$
g^{\varepsilon}(x, t):=z^{\varepsilon}(x)-C+\frac{\gamma t}{4} \exp \left(-\frac{\rho^{+}}{\varepsilon}\right),
$$

and observe that, for each $\varepsilon \in\left(0, \varepsilon_{0}\right), g^{\varepsilon}$ is a subsolution of (2.1).

Let

$$
\tau(\varepsilon)=\frac{4 C}{\gamma} \exp \left(\frac{\rho^{+}}{\varepsilon}\right),
$$

and observe that, for any $(x, t) \in \bar{\Omega} \times[0, \tau(\varepsilon)]$ such that $V_{\alpha}(x)>m$,

$$
g^{\varepsilon}(x, t) \leq z^{\varepsilon}(x) \leq-\exp \left(\frac{r}{\varepsilon}\right) .
$$

We may assume by replacing $\varepsilon_{0}>0$ by a smaller number if necessary that

$$
\exp \left(\frac{r}{\varepsilon_{0}}\right) \geq C \text {. }
$$

Accordingly, we have

$$
g^{\varepsilon} \leq \begin{cases}-\exp \left(\frac{r}{\varepsilon}\right) \leq-C \leq u^{\varepsilon} & \text { in }\left(\bar{\Omega} \backslash \Sigma_{\alpha}^{m}\right) \times[0, T(\varepsilon) \wedge \tau(\varepsilon)), \\ -C \leq u^{\varepsilon}(\cdot, 0) & \text { in } \Sigma_{\alpha}^{m} \times\{0\}, \\ 0 \leq u^{\varepsilon} & \text { in }\left(\Sigma_{\alpha}^{m} \cap \partial \Omega\right) \times(0, T(\varepsilon) \wedge \tau(\varepsilon)) .\end{cases}
$$


Thus,

and, hence, by the comparison principle

$$
g^{\varepsilon} \leq u^{\varepsilon} \text { in } \partial_{\mathrm{p}} Q_{T(\varepsilon) \wedge \tau(\varepsilon)},
$$

$$
g^{\varepsilon} \leq u^{\varepsilon} \text { in } \bar{Q}_{T(\varepsilon) \wedge \tau(\varepsilon)} .
$$

The final step begins by noting that

$$
u^{\varepsilon}(0, \tau(\varepsilon)) \geq g^{\varepsilon}(0, \tau(\varepsilon))=z^{\varepsilon}(0)
$$

and

$$
z^{\varepsilon}(0) \geq-\exp \left(\frac{-m+4 r}{\varepsilon}\right)-\exp \left(\frac{-\rho^{-}}{\varepsilon}\right) \rightarrow 0 \quad \text { as } \varepsilon \rightarrow 0 .
$$

Fix $\delta>0$ and, if necessary, replace $\varepsilon_{0}$ by a smaller number such that $z^{\varepsilon}(0) \geq-\delta$ for all $\varepsilon \in\left(0, \varepsilon_{0}\right)$. Recalling the definition of $\tau(\varepsilon)$ and observing that

$$
u^{\varepsilon}(0, t) \geq z^{\varepsilon}(0) \quad \text { if } t=\frac{4 C}{\gamma} \exp \left(\frac{\rho^{+}}{\varepsilon}\right)<T(\varepsilon) \text { and } 0<\gamma \leq \eta,
$$

we conclude that

$$
u^{\varepsilon}(0, t) \geq-\delta \quad \text { for all } t \in\left[(4 C / \eta) \exp \left(\rho^{+} / \varepsilon\right), T(\varepsilon)\right) \text { and } \varepsilon \in\left(0, \varepsilon_{0}\right) .
$$

Since $\rho^{+}<m-5 r$, by selecting $\varepsilon_{0} \in(0,1)$ sufficiently small, we may assume that $(4 C / \eta) \exp \left(\rho^{+} / \varepsilon\right) \leq \exp (m / \varepsilon)$ for all $\varepsilon \in\left(0, \varepsilon_{0}\right)$, which completes the proof.

5. The proofs of Propositions 2, 3 and 4

Proof of Proposition 2. Since the arguments are similar for both cases when $\beta_{1}<\beta_{2}$ and $\beta_{1}>\beta_{2}$, here we treat only the case $\beta_{1}<\beta_{2}$.

We argue by contradiction and suppose that

$$
\limsup _{k \rightarrow \infty} \lambda_{k}<M\left(\beta_{2}\right) \text {. }
$$

Let $\delta>0$ be a constant to be fixed later, define $\alpha_{\delta}^{+}$and $\mathcal{H}_{\delta}^{+}$as in Section $\left[3\right.$, with $\beta_{0}$ replaced by $\beta_{2}$, and, as in Section 3 , let $V_{\delta}^{+}$be the maximal subsolution of

$$
\mathcal{H}_{\delta}^{+}(x, D u)=0 \quad \text { in } \Omega, \quad u(0)=0,
$$

and set $M_{\delta}^{+}=\min _{\partial \Omega} V_{\delta}^{+}$.

Since Proposition 11 yields

$$
\lim _{\delta \rightarrow 0+} M_{\delta}^{+}=M\left(\beta_{2}\right)
$$

in view of (5.1), we may choose $\delta>0$ so that

$$
\limsup _{k \rightarrow \infty} \lambda_{k}+\delta<M_{\delta}^{+} \text {. }
$$

We fix $m \in \mathbb{R}$ so that

$$
\limsup _{k \rightarrow \infty} \lambda_{k}+\delta<m<M_{\delta}^{+},
$$

and, by passing to a subsequence if necessary, we may assume that

$$
\lambda_{k} \leq m-\delta \quad \text { for all } k \in \mathbb{N} .
$$

Set

$$
\Sigma=\left\{x \in \bar{\Omega}: V_{\delta}^{+}(x) \leq m\right\},
$$

and note that $\Sigma$ is a compact subset of $\Omega$. 
In view of the continuity of the map $t \mapsto u^{\varepsilon}(0, t)$, reselecting, if needed, $\beta_{1}, \mu_{k}$ and $\lambda_{k}$, we may assume that, for all $t \in\left[\exp \left(\mu_{k} / \varepsilon_{k}\right), \exp \left(\lambda_{k} / \varepsilon_{k}\right)\right]$ and $k \in \mathbb{N}$,

$$
\beta_{2}-\frac{\delta}{2}<\beta_{1} \leq u^{\varepsilon_{k}}(0, t) \leq \beta_{2} .
$$

Now we choose $\gamma \in(0, \delta / 2)$ small enough, so that

$$
\Sigma \subset \Omega_{\gamma} \quad \text { and } \quad \beta_{2}-\beta_{1}>2 \gamma .
$$

Proposition 10 gives $\varepsilon_{0} \in(0,1)$ such that, if $\varepsilon \in\left(0, \varepsilon_{0}\right)$,

$$
\left|u^{\varepsilon}(x, t)-u^{\varepsilon}(0, t)\right|<\gamma \quad \text { for all }(x, t) \in \Omega_{\gamma} \times\left[\exp \left(a_{1} / \varepsilon\right), \infty\right) .
$$

We assume that $\varepsilon_{k}<\varepsilon_{0}$ for all $k \in \mathbb{N}$, and combine (5.4) and (5.2), to find

$$
\left|u^{\varepsilon_{k}}(x, t)-\beta_{2}\right| \leq \delta \quad \text { for all }(x, t) \in \Omega_{\gamma} \times\left[\exp \left(\mu_{k} / \varepsilon_{k}\right), \exp \left(\lambda_{k} / \varepsilon_{k}\right)\right],
$$

and

$$
u^{\varepsilon_{k}}\left(x, \exp \left(\mu_{k} / \varepsilon_{k}\right)\right) \leq \beta_{1}+\gamma \quad \text { for all } x \in \Omega_{\gamma} \text { and } k \in \mathbb{N} .
$$

Since (5.5) implies that

$$
a\left(x, u^{\varepsilon_{k}}(x, t)\right) \leq \alpha_{\delta}(x) \text { for all }(x, t) \in \Omega \times\left[\exp \left(\mu_{k} / \varepsilon_{k}\right), \exp \left(\lambda_{k} / \varepsilon_{k}\right)\right], k \in \mathbb{N},
$$

setting

we see that

$$
\left\{\begin{array}{l}
v^{k}(x, t)=u^{\varepsilon_{k}}\left(x, t+\exp \left(\mu_{k} / \varepsilon_{k}\right)\right)-\beta_{1}-\gamma, \\
a^{k}(x, t)=a\left(x, u^{\varepsilon_{k}}\left(x, t+\exp \left(\mu_{k} / \varepsilon_{k}\right)\right)\right),
\end{array}\right.
$$

$$
v_{t}^{k}=\varepsilon_{k} \operatorname{tr}\left[a^{k}(x, t) D^{2} v^{k}\right]+b(x) \cdot D v^{k} \quad \text { for all }(x, t) \in Q .
$$

Furthermore, since $v^{k}(\cdot, 0) \leq 0$ in $\Omega_{\gamma}$, it follows that

$$
v^{k}(\cdot, 0) \leq 0 \text { in } \Sigma .
$$

An application of Proposition [12, with $\varepsilon_{k}, v^{k}$ and $\gamma$ in place of $\varepsilon, u^{\varepsilon}$ and $\delta$ respectively, guarantees that, for sufficiently large $k$, we have

$$
v^{k}(0, t) \leq \gamma \quad \text { for all } t \in\left[0, \exp \left(\lambda_{k} / \varepsilon_{k}\right)-\exp \left(\mu_{k} / \varepsilon_{k}\right)\right],
$$

which, in particular, yields

$$
v^{k}\left(0, \exp \left(\lambda_{k} / \varepsilon_{k}\right)-\exp \left(\mu_{k} / \varepsilon_{k}\right)\right) \leq \gamma .
$$

This shows that

$$
u^{\varepsilon_{k}}\left(0, \exp \left(\lambda_{k} / \varepsilon_{k}\right)\right) \leq \beta_{1}+2 \gamma<\beta_{2}
$$

which is a contradiction.

Proof of Proposition 3. Since the arguments are similar, here we only consider the case where $\beta_{2}<\beta_{1}$ holds.

We suppose that

$$
G^{-}\left(\beta_{2}\right)>\beta_{2},
$$

and obtain a contradiction.

For a small constant $\delta>0$ to be chosen later, define $\alpha_{\delta}^{-}$and $\mathcal{H}_{\delta}^{-}$as in Section 3 , with $\beta_{0}$ replaced by $\beta_{2}$, let $V_{\delta}^{-}$be the quasi-potential corresponding to $\left(\alpha_{\delta}^{-}, b\right)$, that is the maximal subsolution of

$$
\mathcal{H}_{\delta}^{-}(x, D u)=0 \quad \text { in } \Omega \quad \text { and } \quad u(0)=0 .
$$


and $V^{\beta_{2}}$ the quasi-potential corresponding to the pair $\left(a\left(\cdot, \beta_{2}\right), b\right)$, set

$$
M_{\delta}^{-}=\min _{\partial \Omega} V_{\delta}^{-}, \quad \Gamma_{\delta}^{-}=\arg \min \left(V_{\delta}^{-} \mid \partial \Omega\right) \quad \text { and } \quad \Gamma^{\beta_{2}}=\arg \min \left(V^{\beta_{2}} \mid \partial \Omega\right),
$$

and observe that

$$
G^{-}\left(\beta_{2}\right)=\min _{\Gamma^{\beta_{2}}} g
$$

Due to (5.6), we have

$$
\min _{\Gamma^{\beta_{2}}} g>\beta_{2}
$$

Furthermore, in view of (3.5), we may choose $\delta>0$ so that

$$
\min _{\Gamma_{\delta}^{-}} g>\beta_{2}+\delta
$$

Finally replacing, if necessary, $\beta_{1}, \mu_{k}$ and $\lambda_{k}$ we may assume that

$$
\beta_{1} \geq u^{\varepsilon}(0, t) \geq \beta_{2} \quad \text { for all } t \in\left[\exp \left(\mu_{k} / \varepsilon_{k}\right), \exp \left(\lambda_{k} / \varepsilon_{k}\right)\right], k \in \mathbb{N},
$$

and

$$
\beta_{1}<\beta_{2}+\delta / 2
$$

Since, by the maximum principle, $g_{\min } \leq u^{\varepsilon} \leq g_{\max }$ in $\bar{Q}$, we find that Theorem 10 yields $\varepsilon_{0} \in(0,1)$ such that, if $\varepsilon \in\left(0, \varepsilon_{0}\right)$, then

$$
\left|u^{\varepsilon}-u^{\varepsilon}(0, t)\right|<\delta / 2 \text { in } \Omega_{\delta / 2} \times\left[\exp \left(a_{1} / \varepsilon\right), \infty\right) .
$$

Consequently, if $k \in \mathbb{N}$ is sufficiently large, then $\varepsilon_{k}<\varepsilon_{0}$ and

$$
\left|u^{\varepsilon_{k}}-\beta_{2}\right|<\delta \text { in } \Omega_{\delta / 2} \times\left[\exp \left(\mu_{k} / \varepsilon_{k}\right), \exp \left(\lambda_{k} / \varepsilon_{k}\right)\right]
$$

Henceforth, passing if necessary to a subsequence, we assume that (5.10) holds for all $k \in \mathbb{N}$ and, thus

$$
\alpha_{\delta}^{-}(x) \leq a\left(x, u^{\varepsilon_{k}}(x, t)\right) \quad \text { for all }(x, t) \in \bar{\Omega} \times\left[\exp \left(\mu_{k} / \varepsilon_{k}\right), \exp \left(\lambda_{k} / \varepsilon_{k}\right)\right], k \in \mathbb{N} .
$$

We set $\Pi=\left\{x \in \partial \Omega: g(x)>\beta_{2}+\delta\right\}$ and note that, in view of (5.7), $\Pi$ is an open neighborhood, relative to $\partial \Omega$, of $\Gamma_{\delta}^{-}$and

$$
\left\{x \in \bar{\Omega}: V_{\delta}^{-}(x) \leq M_{\delta}^{-}\right\}=\left\{x \in \Omega: V_{\delta}^{-}(x) \leq M_{\delta}^{-}\right\} \cup \Gamma_{\delta}^{-} \subset \Omega^{\Pi},
$$

and deduce, for $\gamma>0$ sufficiently small,

$$
\left\{x \in \bar{\Omega}: V_{\delta}^{-}(x) \leq M_{\delta}^{-}+\gamma\right\} \subset \Omega_{\gamma}^{\Pi} .
$$

In view of (5.8), we observe that

$$
g>\beta_{2}+\delta>\beta_{1} \text { in } \Pi \text {. }
$$

We fix $\gamma>0$ so that (5.12) and $5 \gamma<\beta_{1}-\beta_{2}$ hold, set

$$
\Sigma:=\left\{x \in \bar{\Omega}: V_{\delta}^{-}(x) \leq M_{\delta}^{-}+\gamma\right\} \subset \Omega_{\gamma}^{\Pi} .
$$

Noting that $\beta_{1}>\beta_{1}-4 \gamma>\beta_{2}$, we select a sequence $\left\{\nu_{k}\right\}$ so that

$$
\left\{\begin{array}{l}
\mu_{k}<\nu_{k}<\lambda_{k}, \quad u^{\varepsilon_{k}}\left(0, \exp \left(\nu_{k} / \varepsilon_{k}\right)\right)=\beta_{1}-3 \gamma \quad \text { for all } k \in \mathbb{N}, \\
\beta_{1} \geq u^{\varepsilon_{k}}(0, t) \geq \beta_{1}-3 \gamma \quad \text { for all } t \in\left[\exp \left(\mu_{k} / \varepsilon_{k}\right), \exp \left(\nu_{k} / \varepsilon_{k}\right)\right], k \in \mathbb{N}
\end{array}\right.
$$

Now we show that, for some $\rho>0$ and sufficiently large $k \in \mathbb{N}$,

$$
\exp \left(\nu_{k} / \varepsilon_{k}\right) \geq \exp \left(\mu_{k} / \varepsilon_{k}\right)+\exp \left(\rho / \varepsilon_{k}\right) \text {. }
$$


For this, similarly to (5.9), we use Proposition [10, to find that, for some $r \in\left(0, r_{0}\right)$ and sufficiently large $k \in \mathbb{N}$,

$$
\left|u^{\varepsilon_{k}}-u^{\varepsilon_{k}}(0, \cdot)\right|<\gamma \text { in } B_{r} \times\left[\exp \left(a_{1} / \varepsilon_{k}\right), \infty\right) .
$$

For every such large $k \in \mathbb{N}$, we set

$$
v^{k}(x, t):=u^{\varepsilon_{k}}\left(x, t+\exp \left(\mu_{k} / \varepsilon_{k}\right)\right)-\beta_{1}+\gamma \quad \text { for } \quad(x, t) \in \bar{Q},
$$

and note that

$$
v^{k}(\cdot, 0) \geq 0 \text { in } B_{r}
$$

We apply Proposition 12, with $\varepsilon, u^{\varepsilon}$ and $\alpha$ replaced respectively by $\varepsilon_{k},-v^{k}$ and $\theta_{0}^{-1} I$, to deduce that, for some $\rho>0$,

$$
-v^{k}(0, t) \leq \gamma \quad \text { for all } t \in\left[0, \exp \left(\rho / \varepsilon_{k}\right)\right],
$$

that is,

$$
u^{\varepsilon_{k}}(0, t) \geq \beta_{1}-2 \gamma \quad \text { for all } t \in\left[\exp \left(\mu_{k} / \varepsilon_{k}\right), \exp \left(\mu_{k} / \varepsilon_{k}\right)+\exp \left(\rho / \varepsilon_{k}\right)\right],
$$

which, in view of the choice of $\nu_{k}$, implies that (5.15) holds for sufficiently large $k \in \mathbb{N}$.

In what follows we may assume by replacing if necessary $\left\{\varepsilon_{k}\right\}$ by a further subsequence that (5.15) is satisfied for some $\rho>0$ and all $k \in \mathbb{N}$. We set

$$
w^{k}(x, t)=u^{\varepsilon_{k}}\left(x, t+\exp \left(\mu_{k} / \varepsilon_{k}\right)\right)-\beta_{1}+3 \gamma \quad \text { for } \quad(x, t) \in \bar{Q}, k \in \mathbb{N},
$$

and note that, in view of (5.14) and (5.13),

$$
\begin{cases}w^{k}(0, t) \geq 0 & \text { for all } t \in\left[0, \exp \left(\nu_{k} / \varepsilon_{k}\right)-\exp \left(\mu_{k} / \varepsilon_{k}\right)\right] \\ w^{k}(x, t)=g(x)-\beta_{1}+3 \gamma \geq 0 & \text { for all }(x, t) \in \Pi \times[0, \infty) .\end{cases}
$$

Recalling (5.15), we apply Theorem 9, with $\varepsilon$ and $u^{\varepsilon}$ replaced by $\varepsilon_{k}$ and $-w_{k}$, to get, for sufficiently large $k$,

$$
-w^{k}\left(x, \exp \left(\nu_{k} / \varepsilon_{k}\right)-\exp \left(\mu_{k} / \varepsilon_{k}\right)\right) \leq \gamma \quad \text { for all } x \in \Omega_{\gamma}^{\Pi},
$$

which reads

$$
u^{\varepsilon_{k}}\left(x, \exp \left(\nu_{k} / \varepsilon_{k}\right)\right) \geq \beta_{1}-4 \gamma \quad \text { for all } x \in \Omega_{\gamma}^{\Pi} .
$$

Finally, for $(x, t) \in \bar{Q}$, we set

$$
z^{k}(x, t)=u^{\varepsilon_{k}}\left(x, t+\exp \left(\nu_{k} / \varepsilon_{k}\right)\right)-\beta_{1}+4 \gamma,
$$

observe that, if $k \in \mathbb{N}$ is sufficiently large, then

$$
z^{k}(\cdot, 0) \geq 0 \text { in } \Sigma \text { and } z^{k}=g-\beta_{1}+4 \gamma \geq 0 \text { in } \Pi \times[0, \infty),
$$

and invoke Proposition 13, to conclude that, for sufficiently large $k \in \mathbb{N}$,

$$
z^{k}\left(0, \exp \left(\lambda_{k} / \varepsilon_{k}\right)-\exp \left(\nu_{k} / \varepsilon_{k}\right)\right) \geq-\gamma
$$

and, hence,

$$
u^{\varepsilon_{k}}\left(0, \exp \left(\lambda_{k} / \varepsilon_{k}\right)\right) \geq \beta_{1}-5 \gamma>\beta_{2}
$$

which is a contradiction. 
Proof of Proposition 4. Since the arguments are similar, we give the proof under the assumption that

$$
G^{-}\left(\beta_{0}\right)>\beta_{0}
$$

We suppose that

$$
\rho_{0}>M\left(\beta_{0}\right)
$$

and obtain a contradiction.

Fix $\delta>0$ and let $\alpha_{\delta}^{-}$and $\mathcal{H}_{\delta}^{-}$as in Section 3 and $V_{\delta}^{-}$and $V^{\beta_{0}}$ be the quasi-potentials corresponding to $\left(\alpha_{\delta}^{-}, b\right)$ and $\left(a\left(\cdot, \beta_{0}\right), b\right)$ respectively, set

$$
M_{\delta}^{-}=\min _{\partial \Omega} V_{\delta}^{-}, \quad \Gamma_{\delta}^{-}=\arg \min \left(V_{\delta}^{-} \mid \partial \Omega\right) \quad \text { and } \quad \Gamma^{\beta_{0}}=\arg \min \left(V^{\beta_{0}} \mid \partial \Omega\right),
$$

and note, in view of Proposition 11, (5.16) and (5.17), that

$$
\liminf _{\delta \rightarrow 0+} \min _{\Gamma_{\delta}^{-}} g \geq \min _{\Gamma^{\beta_{0}}} g=G^{-}\left(\beta_{0}\right)>\beta_{0} \text { and } \lim _{\delta \rightarrow 0+} M_{\delta}^{-}=M\left(\beta_{0}\right)<\rho_{0} .
$$

Choose $\delta>0$ so that

$$
\min _{\Gamma_{\delta}^{-}} g>4 \delta+\beta_{0}
$$

For $m>M_{\delta}^{-}$set

$$
\Sigma^{m}:=\left\{x \in \bar{\Omega}: V_{\delta}^{-}(x) \leq m\right\}
$$

and note that

$$
\limsup _{m \rightarrow M_{\delta}^{-}+0} \Sigma^{m} \cap \partial \Omega=\left\{x \in \bar{\Omega}: V_{\delta}^{-}(x) \leq M_{\delta}^{-}\right\} \cap \partial \Omega=\Gamma_{\delta}^{-} .
$$

Hence, we may choose $m \in\left(M_{\delta}^{-}, \rho_{0}\right]$ so that

$$
\Sigma^{m} \cap \partial \Omega \subset\left\{x \in \partial \Omega: g(x)>\beta_{0}+3 \delta\right\} .
$$

The maximum principle yields that, for all $(x, t) \in Q$ and $\varepsilon \in(0,1), u^{\varepsilon}(x, t) \in I_{g}$., while Theorem 10 implies the existence of $\varepsilon_{0} \in(0,1)$ such that for all $(x, t) \in \Omega_{\delta / 2} \times\left[\exp \left(\left(\rho_{0}-\right.\right.\right.$ $\delta) / \varepsilon), \infty)$ and $\varepsilon \in\left(0, \varepsilon_{0}\right)$

$$
\left|u^{\varepsilon}(x, t)-u^{\varepsilon}(0, t)\right|<\frac{\delta}{2} .
$$

Our assumptions yield $\gamma>0$ and a sequence $\left\{\varepsilon_{k}\right\} \subset\left(0, \varepsilon_{0}\right)$ such that $\lim _{k \rightarrow \infty} \varepsilon_{k}=0$ and, for all $\rho \in\left[\rho_{0}-\gamma, \rho_{0}+\gamma\right] \subset(0, \infty)$ and $k \in \mathbb{N}$,

$$
u^{\varepsilon_{k}}\left(0, \exp \left(\rho / \varepsilon_{k}\right)\right) \in\left[\beta_{0}-\delta / 2, \beta_{0}+\delta / 2\right] .
$$

Hence, if $(x, \rho) \in \Omega_{\delta / 2} \times\left[\rho_{0}-\gamma, \rho_{0}+\gamma\right]$ and $k \in \mathbb{N}$, we get

$$
u^{\varepsilon_{k}}\left(x, \exp \left(\rho / \varepsilon_{k}\right)\right) \in\left(\beta_{0}-\delta, \beta_{0}+\delta\right),
$$

and, moreover,

$$
\alpha_{\delta}^{-}(x) \leq a\left(x, u^{\varepsilon_{k}}\left(x, \exp \left(\rho / \varepsilon_{k}\right)\right)\right.
$$

Set

$$
v^{\varepsilon}(x, t):=u^{\varepsilon}\left(x, t+\exp \left(\left(\rho_{0}-\gamma\right) / \varepsilon\right)\right)-\beta_{0}-3 \delta \text { for all }(x, t) \in Q, \varepsilon \in(0,1),
$$

and

$$
a^{\varepsilon}(x, t):=a\left(x, u^{\varepsilon}\left(x, t+\exp \left(\left(\rho_{0}-\gamma\right) / \varepsilon\right)\right)\right) \text { for all }(x, t) \in Q, \varepsilon \in(0,1),
$$


and observe that, for $T_{k}:=\exp \left(\left(\rho_{0}+\gamma\right) / \varepsilon_{k}\right)-\exp \left(\left(\rho_{0}-\gamma\right) / \varepsilon_{k}\right), v^{\varepsilon}$ is a solution of (2.1) and (1.2),

$$
\alpha_{\delta}^{-}(x) \leq a^{\varepsilon_{k}}(x, t) \quad \text { for all }(x, t) \in \Omega \times\left[0, T_{k}\right], k \in \mathbb{N},
$$

and

$$
v^{\varepsilon_{k}}(x, t)=g(x)-\beta_{0}-3 \delta>0 \quad \text { for all }(x, t) \in \Sigma^{m} \cap \partial \Omega \times\left[0, T_{k}\right]
$$

In view of Corollary 14, we may assume, by passing to a subsequence, that

$$
v^{\varepsilon_{k}}(0, t) \geq-\delta \quad \text { for all } t \in\left[\exp \left(m / \varepsilon_{k}\right), T_{k}\right] \text { and } k \in \mathbb{N} .
$$

Since $T_{k}>\exp \left(m / \varepsilon_{k}\right)$ for sufficiently large $k \in \mathbb{N}$, we find $k \in \mathbb{N}$ such that

$$
v^{\varepsilon_{k}}\left(0, T_{k}\right) \geq-\delta
$$

which yields the contradiction

$$
u^{\varepsilon_{k}}\left(0, \exp \left(\left(\rho_{0}+\gamma\right) / \varepsilon_{k}\right)\right) \geq \beta_{0}+3 \delta .
$$

\section{The PROOF OF THE MAIN THEOREM}

The proof of Theorem 1 is a relatively easy consequence of Propositions 2, 3 and 4 as shown in [6, 8]. For the reader's convenience, we reproduce it here. We begin with two lemmata.

Lemma 5. Assume (1.10) and let $u^{\varepsilon} \in C(\bar{Q}) \cap C^{2,1}(Q)$ be a solution of (1.1) and (1.2). For any $\delta>0$ there exist $\lambda_{0}>0$ and $\varepsilon_{0} \in(0,1)$ such that

$$
\left|u^{\varepsilon}(0, t)-g(0)\right| \leq \delta \quad \text { for all } t \in\left[0, \exp \left(\lambda_{0} / \varepsilon\right)\right] \quad \text { and } \quad \varepsilon \in\left(0, \varepsilon_{0}\right) .
$$

Proof. Let $V \in \operatorname{Lip}(\bar{\Omega})$ be the quasi-potential associated with $\left(\theta_{0}^{-1} I, b\right)$. We choose $m>0$ small enough so that $m<\min _{\partial \Omega} V$ and

$$
\{x \in \Omega: V(x) \leq m\} \subset\{x \in \Omega:|g(x)-g(0)| \leq \delta / 2\} .
$$

Applying Proposition 12, with $a^{\varepsilon}(x, t)=a\left(x, u^{\varepsilon}(x, t)\right)$ and $\alpha(x)=\theta_{0}^{-1} I$ and $u^{\varepsilon}$ replaced by $\pm\left(u^{\varepsilon}-g(0)\right)-\delta / 2$, we get that, for each $\gamma>0$, there is $\varepsilon_{0} \in(0,1)$ such that

$$
\pm\left(u^{\varepsilon}(0, t)-g(0)\right)-\delta / 2 \leq \gamma \quad \text { for all } t \in[0, \exp ((m-\gamma) / \varepsilon)] \quad \text { and } \quad \varepsilon \in\left(0, \varepsilon_{0}\right) .
$$

We fix $\gamma>0$ small enough so that $\gamma<\min \{\delta / 2, m\}$, and we get (6.1) with $\lambda_{0}=m-\gamma$.

Lemma 6. Assume (1.10) and (1.17) and let $\lambda>0$ and, for each $\varepsilon \in(, 1), u^{\varepsilon} \in C(\bar{Q}) \cap$ $C^{2,1}(Q)$ be a solution of (1.1) and (1.2). If $c_{1}>c_{0}$, then

$$
\liminf _{\varepsilon \rightarrow 0+} u^{\varepsilon}(0, \exp (\lambda / \varepsilon)) \geq \bar{c}(\lambda)
$$

and, if $c_{1}<c_{0}$, then

$$
\limsup _{\varepsilon \rightarrow 0+} u^{\varepsilon}(0, \exp (\lambda / \varepsilon)) \leq \bar{c}(\lambda) .
$$

This lemma is exactly the same as [8, Lemma 3.12]. 
Proof. We give only the proof of the first assertion, since the other claim can be proved similarly.

Note that, in view of the definition of $c_{1}$ and the function $\bar{c}, G^{-}(c)>c$ for all $c \in\left[c_{0}, c_{1}\right)$ and $\lambda \neq M(c)$ for all $c \in\left[c_{0}, \bar{c}(\lambda)\right)$. Furthermore, since the function $M$ is continuous, we have $\lambda>M(c)$ for all $c \in\left[c_{0}, \bar{c}(\lambda)\right)$.

We show first that, for any $\rho>0$,

$$
\liminf _{\varepsilon \rightarrow 0+} u^{\varepsilon}(0, \exp (\rho / \varepsilon)) \geq c_{0} .
$$

According to Lemma 5 , there exists $\lambda_{0}>0$ such that, for any $\rho \in\left(0, \lambda_{0}\right]$,

$$
\lim _{\varepsilon \rightarrow 0+} u^{\varepsilon}(0, \exp (\rho / \varepsilon))=c_{0},
$$

which shows that (6.4) holds if $\rho \leq \lambda_{0}$.

Fix any $\rho>\lambda_{0}$ and, to prove (6.4), suppose to the contrary that

$$
\liminf _{\varepsilon \rightarrow 0+} u^{\varepsilon}(0, \exp (\rho / \varepsilon))<c_{0} .
$$

It is easily seen that there exist sequences $\left\{\varepsilon_{k}\right\},\left\{\mu_{k}\right\}$ and $\left\{\lambda_{k}\right\}$ of positive numbers and two constants $\beta_{1}, \beta_{2} \in I_{g}$ such that $\lim _{k \rightarrow \infty} \varepsilon_{k}=0, c_{0}>\beta_{1}>\beta_{2}$, and, for all $k \in \mathbb{N}$,

$$
\lambda_{0}<\mu_{k}<\lambda_{k} \leq \rho, \quad u^{\varepsilon_{k}}\left(0, \exp \left(\mu_{k} / \varepsilon_{k}\right)\right)=\beta_{1} \quad \text { and } \quad u^{\varepsilon_{k}}\left(0, \exp \left(\lambda_{k} / \varepsilon_{k}\right)\right)=\beta_{2} .
$$

Since $G^{-}\left(c_{0}\right)>c_{0}$ and $G^{-}$is lower semicontinuous, we may assume reselecting $\beta_{1}, \beta_{2}$ close enough to $c_{0}$ so that $G^{-}\left(\beta_{2}\right)>\beta_{2}$. This contradicts Proposition $[3$, which proves that (6.4) holds.

To show (6.2), in view of (6.4), we may assume that $\bar{c}(\lambda)>c_{0}$ and suppose that (6.2) is false, that is,

$$
\liminf _{\varepsilon \rightarrow 0+} u^{\varepsilon}(0, \exp (\lambda / \varepsilon))<\bar{c}(\lambda)
$$

which in turn implies together with (6.4) that $\bar{c}(\lambda)>c_{0}$.

We set

$$
\hat{c}(\rho):=\liminf _{\varepsilon \rightarrow 0+} u^{\varepsilon}(0, \exp (\rho / \varepsilon)) \quad \text { for } \rho \in(0, \lambda],
$$

and show, arguing by contradiction, that

$$
\hat{c}\left(\rho_{1}\right) \leq \hat{c}\left(\rho_{2}\right) \quad \text { if } 0<\rho_{1}<\rho_{2} \leq \lambda .
$$

To this end, we suppose to the contrary that there exist $0<\rho_{1}<\rho_{2} \leq \lambda$ such that

$$
\hat{c}\left(\rho_{1}\right)>\hat{c}\left(\rho_{2}\right) .
$$

We may assume that $\hat{c}\left(\rho_{2}\right) \leq \hat{c}(\lambda)$. Indeed, if $\hat{c}\left(\rho_{2}\right)>\hat{c}(\lambda)$, then, replacing $\rho_{2}$ by $\lambda$, we find $\hat{c}\left(\rho_{1}\right)>\hat{c}\left(\rho_{2}\right)$ and $\hat{c}\left(\rho_{2}\right)=\hat{c}(\lambda)$. Now, noting by (6.6) and (6.5) that $c_{0} \leq \hat{c}\left(\rho_{2}\right) \leq$ $\hat{c}(\lambda)<\bar{c}(\lambda)$, we may choose sequences $\left\{\varepsilon_{k}\right\},\left\{\mu_{k}\right\},\left\{\lambda_{k}\right\}$ of positive numbers and constants $\beta_{1}, \beta_{2} \in I_{g}$ such that $\lim _{k \rightarrow \infty} \varepsilon_{k}=0, \bar{c}(\lambda)>\beta_{1}>\beta_{2}>c_{0}$, and, for all $k \in \mathbb{N}$,

$$
u^{\varepsilon_{k}}\left(0, \exp \left(\mu_{k} / \varepsilon_{k}\right)\right)=\beta_{1}, \quad u^{\varepsilon_{k}}\left(0, \exp \left(\lambda_{k} / \varepsilon_{k}\right)\right)=\beta_{2} \quad \text { and } \quad \rho_{1}<\mu_{k}<\lambda_{k} \leq \rho_{2} .
$$

Here and there, for notational simplicity, we use the same symbols $\beta_{i}, \varepsilon_{k}, \mu_{k}$ and $\lambda_{k}$ to denote different quantities in different arguments. Moreover, since $c_{0}<\beta_{2}<\bar{c}(\lambda)$, we have $G^{-}\left(\beta_{2}\right)>\beta_{2}$. Thus, we are in the situation that contradicts Proposition 3 , and we conclude that (6.7) holds.

The last step of our proof is an application of Proposition 4 for a contradiction. 
In view of the monotonicity (6.7), the function $\hat{c}$ has at most countably many discontinuities on $(0, \lambda]$ and, recalling that $c_{0} \leq \hat{c}(\rho)<\bar{c}(\lambda)$ for all $\rho \in(0, \lambda]$ and that $G^{-}(c)>c$ and $M(c)<\lambda$ for all $c \in\left[c_{0}, \bar{c}(\lambda)\right)$, we may choose $\rho_{0} \in(0, \lambda)$ so that $\hat{c}$ is continuous at $\rho_{0}$ and, for $\beta_{0}:=\hat{c}\left(\rho_{0}\right)$,

$$
\rho_{0}>M\left(\beta_{0}\right) .
$$

We fix any $\delta>0$. Since $G^{-}\left(\beta_{0}\right)>\beta_{0}$, in view of the lower semicontinuity of $G^{-}$, we may choose $\delta_{1} \in(0, \delta / 3)$ so that

$$
G^{-}(c)>\beta_{0}+3 \delta_{1} \quad \text { for all } c \in\left[\beta_{0}-3 \delta_{1}, \beta_{0}+3 \delta_{1}\right] .
$$

Moreover the continuity of $\hat{c}$ at $\rho_{0}$ yields $\gamma>0$ such that

$$
\left[\rho_{0}-\gamma, \rho_{0}+\gamma\right] \subset(0, \lambda)
$$

and, for all $\rho \in\left[\rho_{0}-\gamma, \rho_{0}+\gamma\right]$,

$$
\hat{c}(\rho) \in\left[\beta_{0}-\delta_{1}, \beta_{0}+\delta_{1}\right] .
$$

Now, we show that there exists a sequence $\left\{\varepsilon_{k}\right\} \subset(0,1)$ such that $\lim _{k \rightarrow \infty} \varepsilon_{k}=0$ and, for all $\rho \in\left[\rho_{0}-\gamma, \rho_{0}+\gamma\right]$ and $k \in \mathbb{N}$,

$$
u^{\varepsilon_{k}}\left(0, \exp \left(\rho / \varepsilon_{k}\right)\right) \in\left[\beta_{0}-3 \delta_{1}, \beta_{0}+3 \delta_{1}\right] .
$$

Indeed, in view of the definition of $\hat{c}$ and (6.10), we may choose a sequence $\left\{\varepsilon_{k}\right\} \subset(0,1)$ such that $\lim _{k \rightarrow \infty} \varepsilon_{k}=0$ and, for all $k \in \mathbb{N}$,

$$
u^{\varepsilon_{k}}\left(0, \exp \left(\left(\rho_{0}+\gamma\right) / \varepsilon_{k}\right)\right) \in\left[\beta_{0}-2 \delta_{1}, \beta_{0}+2 \delta_{1}\right] .
$$

Since $\hat{c}\left(\rho_{0}-\gamma\right) \geq \beta_{0}-\delta_{1}$, (6.10) gives

$$
\liminf _{k \rightarrow \infty} u^{\varepsilon_{k}}\left(0, \exp \left(\left(\rho_{0}-\gamma\right) / \varepsilon_{k}\right)\right) \geq \beta_{0}-\delta_{1},
$$

and, therefore, by passing to a subsequence if necessary, we may assume that, for all $k \in \mathbb{N}$,

$$
u^{\varepsilon_{k}}\left(0, \exp \left(\left(\rho_{0}-\gamma\right) / \varepsilon_{k}\right)\right) \geq \beta_{0}-2 \delta_{1} .
$$

To complete the proof of (6.11), we need only to show that for infinitely many $k \in \mathbb{N}$ and all $\rho \in\left[\rho_{0}-\gamma, \rho_{0}+\gamma\right]$,

$$
u^{\varepsilon_{k}}\left(0, \exp \left(\rho / \varepsilon_{k}\right)\right) \in\left[\beta_{0}-3 \delta_{1}, \beta_{0}+3 \delta_{1}\right] .
$$

If this is not the case, there exist a subsequence of $\left\{\varepsilon_{k}\right\}$, which we denote again by the same symbol, and a sequence $\left\{\rho_{k}\right\} \subset\left[\rho_{0}-\gamma, \rho_{0}+\gamma\right]$ such that either

$$
u^{\varepsilon_{k}}\left(0, \exp \left(\rho_{k} / \varepsilon_{k}\right)\right)>\beta_{0}+3 \delta_{1} \quad \text { for all } k \in \mathbb{N},
$$

or

$$
u^{\varepsilon_{k}}\left(0, \exp \left(\rho_{k} / \varepsilon_{k}\right)\right)<\beta_{0}-3 \delta_{1} \quad \text { for all } k \in \mathbb{N},
$$

In view of (6.12), if (6.15) holds, then there are two sequences $\left\{\mu_{k}\right\},\left\{\lambda_{k}\right\} \subset(0, \lambda)$ such that, for all $k \in \mathbb{N}$,

$$
\left\{\begin{array}{l}
\beta_{0}+3 \delta_{1}=u^{\varepsilon_{k}}\left(0, \exp \left(\mu_{k} / \varepsilon_{k}\right)\right)>u^{\varepsilon_{k}}\left(0, \exp \left(\lambda_{k} / \varepsilon_{k}\right)\right)=\beta_{0}+2 \delta_{1}, \\
\rho_{0}-\gamma \leq \mu_{k}<\lambda_{k} \leq \rho_{0}+\gamma .
\end{array}\right.
$$


Similarly, in view of (6.13), if (6.16) holds, then there are two sequences $\left\{\mu_{k}\right\},\left\{\lambda_{k}\right\} \subset$ $(0, \lambda)$ such that, for all $k \in \mathbb{N}$,

$$
\left\{\begin{array}{l}
\beta_{0}-2 \delta_{1}=u^{\varepsilon_{k}}\left(0, \exp \left(\mu_{k} / \varepsilon_{k}\right)\right)>u^{\varepsilon_{k}}\left(0, \exp \left(\lambda_{k} / \varepsilon_{k}\right)\right)=\beta_{0}-3 \delta_{1}, \\
\rho_{0}-\gamma \leq \mu_{k}<\lambda_{k}<\rho_{0}+\gamma .
\end{array}\right.
$$

If (6.17) holds, setting $\beta_{1}:=\beta_{0}+3 \delta_{1}$ and $\beta_{2}:=\beta_{0}+2 \delta_{1}$ and noting by (6.9) that $G^{-}\left(\beta_{2}\right)>\beta_{2}$, we apply Proposition 3 , to obtain a contradiction.

In the case (6.18) holds, setting $\beta_{1}:=\beta_{0}-2 \delta_{1}$ and $\beta_{2}:=\beta_{0}-3 \delta_{1}$ and noting that $G^{-}\left(\beta_{2}\right)>\beta_{2}$, we get a contradiction by Proposition 3 ,

Now, we find that (6.14) holds and, therefore, there is a sequence $\left\{\varepsilon_{k}\right\} \subset(0,1)$ for which (6.11) holds.

Thus, under the supposition (6.6), we have shown that (6.8) and (6.10) hold for some sequence $\left\{\varepsilon_{k}\right\} \subset(0,1)$ converging to zero. Proposition 4 assures that $\rho_{0} \leq M\left(\beta_{0}\right)$, which contradicts (6.8). Therefore, we conclude that (6.2) must hold.

Proof of Theorem [1. In view of Theorem 10, we only need to show that

$$
\lim _{\varepsilon \rightarrow 0} u^{\varepsilon}(0, \exp (\lambda / \varepsilon))=\bar{c}(\lambda) \text {. }
$$

The comparison principle yields that

$$
g_{\min } \leq u^{\varepsilon} \leq g_{\max } \text { on } \bar{Q} .
$$

We fix $\lambda>0$ and consider first the case $\lambda<M\left(c_{0}\right)$, which implies that $\bar{c}(\lambda)=c_{0}$, and prove that

$$
\limsup _{\varepsilon \rightarrow 0} u^{\varepsilon}(0, \exp (\lambda / \varepsilon)) \leq \bar{c}(\lambda)=c_{0} .
$$

We argue by contradiction and suppose that

$$
\limsup _{\varepsilon \rightarrow 0} u^{\varepsilon}(0, \exp (\lambda / \varepsilon))>c_{0} .
$$

Using the continuity of the function $M$, we choose $\beta_{1}, \beta_{2} \in \mathbb{R}$ so that

$$
c_{0}<\beta_{1}<\beta_{2}<\limsup _{\varepsilon \rightarrow 0} u^{\varepsilon}(0, \exp (\lambda / \varepsilon)) \text { and } M\left(\beta_{2}\right)>\lambda,
$$

and note that, in view of Lemma 5, there are constants $\lambda_{0} \in(0, \lambda)$ and $\varepsilon_{0} \in(0,1)$ such that

$$
u^{\varepsilon}\left(0, \exp \left(\lambda_{0} / \varepsilon\right)\right) \leq \beta_{1} \quad \text { for all } \varepsilon \in\left(0, \varepsilon_{0}\right) .
$$

On the other hand, (6.21) yields a sequence $\left\{\varepsilon_{k}\right\}_{k \in \mathbb{N}} \subset\left(0, \varepsilon_{0}\right)$ such that $\varepsilon_{k} \rightarrow 0$ and

$$
u^{\varepsilon_{k}}\left(0, \exp \left(\lambda / \varepsilon_{k}\right)\right) \geq \beta_{2} \quad \text { for all } k \in \mathbb{N},
$$

while, (6.22) gives

$$
u^{\varepsilon_{k}}\left(0, \exp \left(\lambda_{0} / \varepsilon_{k}\right)\right) \leq \beta_{1} \quad \text { for all } k \in \mathbb{N} .
$$

The continuity of $t \mapsto u^{\varepsilon_{k}}(0, t)$ implies that, for each $k \in \mathbb{N}$, there exist $\mu_{k}, \lambda_{k} \in\left[\lambda_{0}, \lambda\right]$ such that $\lambda_{0} \leq \mu_{k}<\lambda_{k} \leq \lambda$ and

$$
u^{\varepsilon_{k}}\left(0, \exp \left(\mu_{k} / \varepsilon_{k}\right)\right)=\beta_{1} \quad \text { and } \quad u^{\varepsilon_{k}}\left(0, \exp \left(\lambda_{k} / \varepsilon_{k}\right)\right)=\beta_{2} .
$$

Proposition 2 now assures that $\lim _{\sup _{k \rightarrow \infty}} \lambda_{k} \geq M\left(\beta_{2}\right)$, but this contradicts that $\lambda_{k} \leq$ $\lambda<M\left(\beta_{2}\right)$ for all $k \in \mathbb{N}$. 
A similar argument shows that

$$
\liminf _{\varepsilon \rightarrow 0} u^{\varepsilon}(0, \exp (\lambda / \varepsilon)) \geq \bar{c}(\lambda),
$$

and, thus, we have (6.19) in the case where $\lambda<M\left(c_{0}\right)$.

Next we consider the case where $\lambda \geq M\left(c_{0}\right)$ and $c_{1}=c_{0}$ and recall that, by definition, $\bar{c}(\lambda)=c_{0}$. We first suppose that

$$
\limsup _{\varepsilon \rightarrow 0} u^{\varepsilon}(0, \exp (\lambda / \varepsilon))>c_{0},
$$

and use (1.15) and the upper semicontinuity of $G^{+}$, to select $\beta_{2} \in \mathbb{R}$ so that $c_{0}<\beta_{2}<$ $\limsup _{\varepsilon \rightarrow 0} u^{\varepsilon}(0, \exp (\lambda / \varepsilon))$ and $G^{+}\left(\beta_{2}\right)<\beta_{2}$.

Choosing, for instance, $\beta_{1}=\left(c_{0}+\beta_{2}\right) / 2$, so that $c_{0}<\beta_{1}<\beta_{2}$, and, using Lemma 5 as in the previous case, we may choose sequences $\left\{\varepsilon_{k}\right\},\left\{\mu_{k}\right\},\left\{\lambda_{k}\right\}$ such that $\lim _{k \rightarrow \infty} \varepsilon_{k}=0$ and for some $\lambda_{0}>0$ and all $k \in \mathbb{N}$,

$$
\lambda_{0} \leq \mu_{k}<\lambda_{k} \leq \lambda, \quad u^{\varepsilon_{k}}\left(0, \exp \left(\mu_{k} / \varepsilon_{k}\right)\right)=\beta_{1} \quad \text { and } \quad u^{\varepsilon_{k}}\left(0, \exp \left(\lambda_{k} / \varepsilon_{k}\right)\right)=\beta_{2} .
$$

This contradicts Proposition [3, and thus, we conclude that

$$
\limsup _{\varepsilon \rightarrow 0} u^{\varepsilon}(0, \exp (\lambda / \varepsilon)) \leq c_{0} .
$$

A similar argument shows

$$
\liminf _{\varepsilon \rightarrow 0} u^{\varepsilon}(0, \exp (\lambda / \varepsilon)) \geq c_{0}
$$

and, hence, we have (6.19) when $\lambda \geq M\left(c_{0}\right)$ and $c_{1}=c_{0}$.

Now we consider the case where $\lambda \geq M\left(c_{0}\right)$ and $c_{1}>c_{0}$. The definition of $c_{1}$ implies that $G^{-}(c)>c$ for all $c \in\left[c_{0}, c_{1}\right)$, and, moreover, by the definition of $\bar{c}$, we have $\bar{c}(\lambda) \in\left[c_{0}, c_{1}\right]$, $\lambda>M(c)$ for all $c \in\left[c_{0}, \bar{c}(\lambda)\right)$, and, if $\bar{c}(\lambda)<c_{1}$, then $M(\bar{c}(\lambda))=\lambda$.

Suppose that

$$
\limsup _{\varepsilon \rightarrow 0} u^{\varepsilon}(0, \exp (\lambda / \varepsilon))>\bar{c}(\lambda) .
$$

We assume first that $\bar{c}(\lambda)=c_{1}$ and observe that we must have $c_{1}<g_{\max }$. Then (1.15) yields $\beta_{2} \in \mathbb{R}$ so that $G^{+}\left(\beta_{2}\right)<\beta_{2}$ and $c_{1}<\beta_{2}<\lim \sup _{\varepsilon \rightarrow 0} u^{\varepsilon}(0, \exp (\lambda / \varepsilon))$. Fixing $\beta_{1} \in\left(c_{1}, \beta_{2}\right)$, we argue, as in the previous case, with $c_{1}$ in place of $c_{0}$ and find sequences $\varepsilon_{k} \rightarrow 0+,\left\{\mu_{k}\right\}$ and $\left\{\lambda_{k}\right\}$, and constants $\lambda_{0}>0$ and $\delta>0$ such that for all $k \in \mathbb{N}$,

$$
\lambda_{0} \leq \mu_{k}<\lambda_{k} \leq \lambda, \quad u^{\varepsilon_{k}}\left(0, \exp \left(\mu_{k} / \varepsilon_{k}\right)=\beta_{1}, \quad u^{\varepsilon_{k}}\left(0, \exp \left(\lambda_{k} / \varepsilon_{k}\right)\right)=\beta_{2},\right.
$$

which contradicts Proposition 3 ,

Assume next that $\bar{c}(\lambda)<c_{1}$. As noted above, we have $M(\bar{c}(\lambda))=\lambda$ and $M(c)<\lambda$ for all $c \in\left[c_{0}, \bar{c}(\lambda)\right)$, and, in particular,

$$
M(c) \leq \lambda \text { for all } c \in\left[c_{0}, \bar{c}(\lambda)\right]
$$

Since the function $\bar{c}$ is continuous at $\lambda$, we may choose $\eta>0$ so that $\bar{c}(r)<c_{1}$ for all $r \in[\lambda, \lambda+\eta]$ and noting that, for any $r \in(\lambda, \lambda+\eta], r>M\left(c_{0}\right)$, we find by the definition of $\bar{c}(r)$ that $M(\bar{c}(r))=r$, which together with (6.23) implies that $\bar{c}(r)>\bar{c}(\lambda)$.

We choose $\gamma \in(0, \eta)$ small enough so that $\bar{c}(\lambda+\gamma)<\lim _{\sup _{\varepsilon \rightarrow 0}} u^{\varepsilon}(0, \exp (\lambda / \varepsilon))$. If we set $\beta_{2}=\bar{c}(\lambda+\gamma)$ and fix $\beta_{1} \in\left(\bar{c}(\lambda), \beta_{2}\right)$, then we have $\bar{c}(\lambda)<\beta_{1}<\beta_{2}<$ $\lim \sup _{\varepsilon \rightarrow 0} u^{\varepsilon}(0, \exp (\lambda / \varepsilon))$. 
As before, we choose sequences $\left\{\varepsilon_{k}\right\},\left\{\mu_{k}\right\}$ and $\left\{\lambda_{k}\right\}$ such that $\lim _{k \rightarrow \infty} \varepsilon_{k}=0$ and, for some $\lambda_{0}>0$ and for all $k \in \mathbb{N}$,

$$
\lambda_{0} \leq \mu_{k}<\lambda_{k} \leq \lambda, \quad u^{\varepsilon_{k}}\left(0, \exp \left(\mu_{k} / \varepsilon_{k}\right)\right)=\beta_{1} \text { and } u^{\varepsilon_{k}}\left(0, \exp \left(\lambda_{k} / \varepsilon_{k}\right)\right)=\beta_{2} .
$$

Then Proposition 2 imply that $M\left(\beta_{2}\right) \leq \lim \sup _{k \rightarrow \infty} \lambda_{k} \leq \lambda$. On the other hand, we have $M\left(\beta_{2}\right)=M(\bar{c}(\lambda+\gamma))=\lambda+\gamma>\lambda$. Hence we obtain a contradiction,

Thus, in the case when $\lambda \geq M\left(c_{0}\right)$ and $c_{1}>c_{0}$, we have

$$
\limsup _{\varepsilon \rightarrow 0} u^{\varepsilon}(0, \exp (\lambda / \varepsilon)) \leq \bar{c}(\lambda)
$$

while, by Lemma 6, we find

$$
\liminf _{\varepsilon \rightarrow 0} u^{\varepsilon}(0, \exp (\lambda / \varepsilon)) \geq \bar{c}(\lambda)
$$

and we conclude that (6.19) holds when $\lambda \geq M\left(c_{0}\right)$ and $c_{1}>c_{0}$.

A similar argument proves that (6.19) holds when $\lambda \geq M\left(c_{0}\right)$ and $c_{1}<c_{0}$, and the proof is complete.

\section{Appendix A. A subsolution PROperty}

For $T>0$ and a (relatively) open subset $\Pi$ of $\partial \Omega$, we consider the problem

$$
\left\{\begin{array}{l}
U_{t} \leq b(x) \cdot D U \quad \text { in } \quad \Omega \times(0, T], \\
\min \left\{U_{t}-b(x) \cdot D U, U\right\} \leq 0 \quad \text { on } \quad \Pi \times(0, T] .
\end{array}\right.
$$

Lemma A.1. Let $U \in \operatorname{USC}\left(\bar{Q}_{T}\right)$ be a subsolution of (A.1), fix $z \in \Omega^{\Pi}$ and set

$$
u(t)=U(X(T-t, z), t) \quad \text { for } t \in[0, T] .
$$

Then $u \in \operatorname{USC}([0, T])$ and, if $z \in \Omega$, it is a subsolution of

$$
u^{\prime} \leq 0 \quad \text { in }(0, T]
$$

and, if $z \in \Pi$, it is a subsolution of

$$
\left\{\begin{array}{l}
u^{\prime} \leq 0 \text { in }(0, T) \\
\min \left\{u^{\prime}, u\right\} \leq 0 \text { on }\{T\}
\end{array}\right.
$$

We note that observations like the lemma above concerning the restriction of viscosity solutions to lower dimensional manifolds go back to Crandall and Lions [4, Proposition I.13].

Proof. Let $\phi \in C^{1}((0, T])$ and assume that $u-\phi$ has a strict maximum at $\hat{t} \in(0, T]$.

For $\alpha>0$ consider the function $\Phi: \bar{Q}_{T} \rightarrow \mathbb{R}$ given by

$$
\Phi(x, t):=U(x, t)-\phi(t)-\alpha|x-X(T-t, z)|^{2},
$$

let $\left(x_{\alpha}, t_{\alpha}\right) \in \bar{Q}_{T}$ be a maximum point of $\Phi$, set $\hat{x}=X(T-\hat{t}, z)$, and observe that, as $\alpha \rightarrow \infty,\left(x_{\alpha}, t_{\alpha}\right) \rightarrow(\hat{x}, \hat{t}), \alpha\left|x_{\alpha}-X\left(T-t_{\alpha}, z\right)\right|^{2} \rightarrow 0$ and $U\left(x_{\alpha}, t_{\alpha}\right) \rightarrow U(\hat{x}, \hat{t})$.

Then, for $\alpha$ sufficiently large, we may assume that $\left(x_{\alpha}, t_{\alpha}\right) \in \Omega \times(0, T]$ if either $z \in \Omega$ or $\hat{t}<T$, and $\left(x_{\alpha}, t_{\alpha}\right) \in \Omega^{\Pi} \times(0, T]$ if $z \in \Pi$.

If $\left(x_{\alpha}, t_{\alpha}\right) \in \Omega \times(0, T]$, A.1 yields

$$
\phi^{\prime}\left(t_{\alpha}\right)-2 \alpha\left(X\left(T-t_{\alpha}, z\right)-x_{\alpha}\right) \cdot \dot{X}\left(T-t_{\alpha}, z\right) \leq 2 \alpha b\left(x_{\alpha}\right) \cdot\left(x_{\alpha}-X\left(T-t_{\alpha}, z\right)\right),
$$


and then

$$
\begin{aligned}
\phi^{\prime}\left(t_{\alpha}\right) & \leq 2 \alpha\left(x_{\alpha}-X\left(T-t_{\alpha}, z\right)\right) \cdot\left(b\left(x_{\alpha}\right)-b\left(X\left(T-t_{\alpha}, z\right)\right)\right) \\
& \left.\leq 2\|D b\|_{L^{\infty}(\Omega)} \alpha \mid x_{\alpha}-X\left(T-t_{\alpha}, z\right)\right)\left.\right|^{2} .
\end{aligned}
$$

Similarly, if $\left(x_{\alpha}, t_{\alpha}\right) \in \Pi \times(0, T]$, then we get

$$
\left.\phi^{\prime}\left(t_{\alpha}\right) \leq 2\|D b\|_{L^{\infty}(\Omega)} \alpha \mid x_{\alpha}-X\left(T-t_{\alpha}, z\right)\right)\left.\right|^{2} \quad \text { or } \quad U\left(x_{\alpha}, t_{\alpha}\right) \leq 0 .
$$

Sending $\alpha \rightarrow \infty$ yields

$$
\phi^{\prime}(\hat{t}) \leq 0 \quad \text { if either } z \in \Omega \text { or } \hat{t}<T
$$

and

$$
\phi^{\prime}(\hat{t}) \leq 0 \quad \text { or } \quad u(\hat{t}) \leq 0 \quad \text { if } \quad z \in \Pi \text { and } \hat{t}=T .
$$

\section{Appendix B. The supersolution Property up to the BOUndary}

For $\alpha \in C\left(\bar{\Omega}, \mathbb{S}^{n}\left(\theta_{0}\right)\right)$ and $H(x, p)=\alpha(x) p \cdot p+b(x) \cdot p$ we consider the equation

$$
H(x, D u)=0 \quad \text { in } \Omega .
$$

Lemma B.1. The maximal subsolution $V \in \operatorname{Lip}(\bar{\Omega})$ of (B.1) with $V(0)=0$ satisfies, in the viscosity sense,

$$
H(x, D V) \geq 0 \quad \text { on } \bar{\Omega} .
$$

Note that the importance of the lemma above is that the viscosity inequality holds up to the boundary.

Proof. Let $\phi \in C^{1}(\bar{\Omega})$ and assume that $V-\phi$ has a strict minimum at $\hat{x} \in \bar{\Omega}$ and $V(\hat{x})=$ $\phi(\hat{x})$.

To prove the assertion of the lemma, we argue by contradiction and suppose that $H(\hat{x}, D \phi(\hat{x}))<$ 0 .

Indeed, if $\hat{x}=0$, then

$$
H(\hat{x}, D \phi(\hat{x}))=\alpha(0) D \phi(0) \cdot D \phi(0) \geq 0,
$$

and, henceforth, we may assume that $\hat{x} \neq 0$.

We may choose constants $r>0$ and $\varepsilon>0$ so that $0 \notin B_{r}(\hat{x})$ and

$$
\begin{array}{ll}
H(x, D \phi(x)) \leq 0 & \text { for all } x \in \bar{\Omega} \cap B_{r}(\hat{x}), \\
\varepsilon+\phi(x)<V(x) & \text { for all } x \in \bar{\Omega} \backslash B_{r}(\hat{x}) .
\end{array}
$$

It follows from (B.2) that, in the viscosity sense,

$$
H(x, D \phi) \leq 0 \quad \text { in } \Omega \cap B_{r}(\hat{x}) .
$$

Set

$$
W(x)=\max \{V(x), \varepsilon+\phi(x)\} \quad \text { for } \quad x \in \bar{\Omega},
$$

and observe that $\Omega=N \cup M$, where $N:=\Omega \cap B_{r}(\hat{x})$ and $M:=\{x \in \Omega: V(x)>\varepsilon+\phi(x)\}$ (note that $N, M$ are both open subsets of $\Omega$ ),

$$
H(x, D W) \leq 0 \quad \text { in } N \text { in the viscosity sense, }
$$

$W=V$ in $M$ and $\hat{x} \in M$. Hence, $W$ is a subsolution of (B.1) such that $W(0)=V(0)=0$ and $W(\hat{x})>V(\hat{x})$, which contradicts the maximality of $V$. 


\section{ApPendix C. A COMPARISON THEOREM}

We follow the arguments of [10, Corollary $2.2 \&$ Remark 2.4] to give a proof of following lemma.

Lemma C.1. Let $\alpha \in C\left(\mathbb{R}^{n}, \mathbb{S}^{n}\left(\theta_{0}\right)\right)$ and $H(x, p)=\alpha(x) p \cdot p+b(x) \cdot p$. If $v \in \operatorname{Lip}(\bar{\Omega})$ and $w \in \operatorname{LSC}(\bar{\Omega})$ are respectively a subsolution and a supersolution of the state-constraints problem

$$
H(x, D u)=0 \quad \text { in } \Omega,
$$

that is, $v$ and $w$ satisfy, respectively,

$$
H(x, D v) \leq 0 \quad \text { in } \Omega \quad \text { and } \quad H(x, D w) \geq 0 \quad \text { on } \bar{\Omega},
$$

and $v(0) \leq w(0)$, then $u \leq v$ on $\bar{\Omega}$.

Note that the viscosity property of $v$ and $w$ at the origin is indeed not required in the lemma above. That is, it is enough to assume that $v$ and $w$ are a subsolution of

$$
H(x, D v) \leq 0 \quad \text { in } \Omega \backslash\{0\},
$$

and a supersolution of

$$
H(x, D w) \geq 0 \quad \text { on } \quad \bar{\Omega} \backslash\{0\} .
$$

Proof. Fix $\varepsilon>0$ and choose $r \in\left(0, r_{0}\right)$ sufficiently small so that

$$
\max _{\partial B_{r}} v \leq \min _{\partial B_{r}} w+\varepsilon
$$

set $\Omega(r):=\Omega \backslash \bar{B}_{r}$, define $h \in C(\partial \Omega(r))$ and $v_{\varepsilon} \in \operatorname{Lip}(\bar{\Omega})$ by

$$
v_{\varepsilon}=v-\varepsilon \text { and } h(x)= \begin{cases}\min _{\partial B_{r}} w & \text { if } x \in \partial B_{r}, \\ \max _{\partial \Omega} v & \text { if } x \in \partial \Omega,\end{cases}
$$

and observe that $v_{\varepsilon}$ and $w$ are, respectively, a subsolution and a supersolution of the Dirichlet problem in the viscosity sense (see [10]):

$$
\left\{\begin{array}{l}
H(x, D u)=0 \quad \text { in } \Omega(r), \\
u=h \quad \text { or } \quad H(x, D u)=0 \quad \text { on } \partial \Omega(r) .
\end{array}\right.
$$

It follows from [11, Corollary 4] that there exists $\psi \in \operatorname{Lip}(\bar{\Omega}(r))$ which is a subsolution of $H(x, D \psi) \leq-\eta$ in $\Omega(r)$ for some $\eta>0$ and note that we may assume by adding, if necessary, a constant that $\psi \leq v_{\varepsilon}$ on $\Omega(r)$.

Define $v^{\varepsilon} \in \operatorname{Lip}(\bar{\Omega}(r))$ by $v^{\varepsilon}(x)=(1-\varepsilon) v_{\varepsilon}(x)+\varepsilon \psi(x)$ and note that $v^{\varepsilon}$ is a subsolution of

$$
\left\{\begin{array}{l}
H(x, D u) \leq-\varepsilon \eta \quad \text { in } \Omega(r), \\
u \leq h \quad \text { or } \quad H(x, D u) \leq-\varepsilon \eta \quad \text { on } \partial \Omega(r) .
\end{array}\right.
$$

It is clear that the domain $\Omega(r)$ satisfies the uniform interior cone condition and, hence, we apply [10, Corollary $2.2 \&$ Remark 2.4] to $v^{\varepsilon}$ and $w_{\varepsilon}$, to conclude that $v^{\varepsilon} \leq w_{\varepsilon}$ in $\bar{\Omega}(r)$, from which, after sending $\varepsilon \rightarrow 0$, we get $v \leq w$ on $\bar{\Omega}$. 


\section{REFERENCES}

[1] Martino Bardi and Italo Capuzzo-Dolcetta, Optimal control and viscosity solutions of Hamilton-JacobiBellman equations, Systems \& Control: Foundations \& Applications, Birkhäuser Boston, Inc., Boston, MA, 1997. With appendices by Maurizio Falcone and Pierpaolo Soravia. MR1484411 (99e:49001)

[2] Guy Barles, Solutions de viscosité des équations de Hamilton-Jacobi, Mathématiques \& Applications (Berlin) [Mathematics \& Applications], vol. 17, Springer-Verlag, Paris, 1994 (French, with French summary). MR1613876 (2000b:49054)

[3] Michael G. Crandall, Hitoshi Ishii, and Pierre-Louis Lions, User's guide to viscosity solutions of second order partial differential equations, Bull. Amer. Math. Soc. (N.S.) 27 (1992), no. 1, 1-67, DOI 10.1090/S0273-0979-1992-00266-5. MR1118699 (92j:35050)

[4] Michael G. Crandall and Pierre-Louis Lions, Viscosity solutions of Hamilton-Jacobi equations, Trans. Amer. Math. Soc. 277 (1983), no. 1, 1-42, DOI 10.2307/1999343. MR690039 (85g:35029)

[5] Wendell H. Fleming and H. Mete Soner, Controlled Markov processes and viscosity solutions, 2nd ed., Stochastic Modelling and Applied Probability, vol. 25, Springer, New York, 2006. MR2179357 (2006e:93002)

[6] M. Freidlin and L. Koralov, Nonlinear stochastic perturbations of dynamical systems and quasi-linear parabolic PDE's with a small parameter, Probab. Theory Related Fields 147 (2010), no. 1-2, 273-301, DOI 10.1007/s00440-009-0208-8. MR2594354 (2011c:60085)

[7] , Metastability for nonlinear random perturbations of dynamical systems, arXiv:0903.0430v2 (2012), 1-23.

[8] Nonlinear stochastic perturbations of dynamical systems and quasi-linear parabolic PDEfs with a small parameter, ArXiv:0903.0428v2 (2012), 1-29.

[9] Mark I. Freidlin and Alexander D. Wentzell, Random perturbations of dynamical systems, 3rd ed., Grundlehren der Mathematischen Wissenschaften [Fundamental Principles of Mathematical Sciences], vol. 260, Springer, Heidelberg, 2012. Translated from the 1979 Russian original by Joseph Szücs. MR2953753

[10] Hitoshi Ishii, A boundary value problem of the Dirichlet type for Hamilton-Jacobi equations, Ann. Scuola Norm. Sup. Pisa Cl. Sci. (4) 16 (1989), no. 1, 105-135. MR1056130 (91f:35071)

[11] Hitoshi Ishii and Panagiotis E. Souganidis, Metastability for parabolic equations with drift: Part 1, Indiana Univ. Math. J. 64 (2015), no. 3, 875-913, DOI 10.1512/iumj.2015.64.5559.

[12] N. V. Krylov, Nonlinear elliptic and parabolic equations of the second order, Mathematics and its Applications (Soviet Series), vol. 7, D. Reidel Publishing Co., Dordrecht, 1987. Translated from the Russian by P. L. Buzytsky [P. L. Buzytski1]. MR901759 (88d:35005)

[13] Pierre-Louis Lions, Generalized solutions of Hamilton-Jacobi equations, Research Notes in Mathematics, vol. 69, Pitman (Advanced Publishing Program), Boston, Mass.-London, 1982. MR667669 (84a:49038)

[14] Halil Mete Soner, Optimal control with state-space constraint. I, SIAM J. Control Optim. 24 (1986), no. 3, 552-561, DOI 10.1137/0324032. MR838056 (87e:49029)

E-mail address: hitoshi.ishii@waseda.jp (Hitoshi Ishii), souganidis@math.uchicago.edu (Panagiotis

E. Souganidis) 